\title{
Instability of wind-forced inertial oscillations
}

\author{
By A. M. TREGUIER AND P. KLEIN \\ Laboratoire de Physique des Océans, IFREMER, Centre de Brest, BP70, 29280 Piouzané, France
}

(Received 2 September 1993 and in revised form 11 February 1994)

An instability mechanism that can amplify wind-forced inertial oscillations in the upper ocean is investigated. This forced instability happens because of the phase relationship between the mixed-layer depth and the surface current. It allows the inertial oscillations propagating against the wind to extract energy from it and amplify. The key ingredients for the instability to work are $(a)$ a non-zero mean wind stress, $(b)$ a spatial variability of the oscillations in the direction of the wind stress. The amplification is demonstrated using a simple shallow-water model in a few situations: the dispersion of a localized disturbance with steady and time-varying wind forcing, generation of inertial waves at a coast, and spatial variability induced by mesoscale eddies. Estimates of the growth rate are provided for both dissipative and nondissipative cases.

\section{Introduction}

The surface layer of the ocean is generally well mixed in temperature and salinity over a depth of 10-100 m. In this mixed layer, currents are often found to oscillate with nearly the local inertial (Coriolis) frequency. There is a strong observational evidence of correlation between those energetic inertial oscillations and local intense wind events, starting from Day \& Webster (1965). It is thus believed that inertial oscillations are mainly wind-driven, although the possibility of over-reflection of internal waves from below the mixed layer has been examined (Kamachi \& Grimshaw 1984). The linear theory of inertial wave generation by the wind and the radiation of this energy at depth is now well understood (see for example Pollard 1970 or Gill 1984). Models have been compared with observations by Pollard \& Millard (1970), Price (1981), and others.

Nonlinear effects have been neglected in all those studies because it was assumed that the spatial scale $L$ of the inertial oscillations was comparable with the wind stress scale (hundreds of kilometres) and therefore the advective timescale $L / U$ was large compared with the inertial period.

In situ data of the mixed layer do not support that view. Starting at the end of the seventies, observations have revealed an unexpected variability of the inertial oscillations on spatial scales of order $50 \mathrm{~km}$ or less, much smaller than the scales of the local winds. Weller (1982) reported such observations made during the JASIN experiment. He suggested that the spatial variability between two moorings $44 \mathrm{~km}$ apart was due to the presence of a mesoscale eddy field. Indeed, simple linearized theory shows that the divergence of an eddy field can make inertial oscillations grow or decay, whereas the local vorticity shifts the apparent inertial frequency. Similar observations have been made recently during the FASINEX experiment (Weller et al. 1991).

The small spatial scale of the inertial motions warrants further investigation of the role of nonlinear terms in their dynamics. Nonlinear phenomena affecting the 
propagation of internal waves are extensively covered in the literature: wave-wave and wave-vortical mode interactions (Lelong \& Riley 1991; Grimshaw 1988), internal solitons (Renouard, Chabert d'Hières \& Zhang 1987), breaking and critical layers. On the other hand, the way nonlinearity affects the generation of near inertial waves by the wind has seldom been considered. It is the topic of the present paper.

Recently Klein \& Treguier (1993, hereinafter referred to as KT93) studied the oceanic response to a steady wind stress when a geostrophic jet is present. They found that the wind-driven inertial oscillations in the mixed layer can amplify in some cases owing to a resonance phenomenon present in the nonlinear equations. The energy generated that way can easily propagate in the ocean interior, leading to a significant increase of the internal wave activity and of the mixing processes. In the present paper, we demonstrate that the mechanism invoked by KT93 is a much more general phenomenon: it is a forced instability mechanism that requires only the presence of a non-zero mean wind stress and the existence of a spatial variability in the inertial wave field. This mechanism is described in $\$ 2$. In $\$ 3$ we explore its robustness for twodimensional coastal flows and time-varying wind forcings. Then, in $\$ 4$, we extend the results of KT93 to two-dimensional geostrophic eddies.

\section{The instability mechanism}

The simplest model for a study of the wind-forced inertial oscillations is the 'shallow-water' model. In this approximation, the upper ocean is made of an active layer with reference uniform depth $h_{0}$ at rest, lying over an infinitely deep (motionless) bottom layer. The active layer is vertically well-mixed with uniform density and horizontal velocities $u$ and $v$. The equations on an $f$-plane, in Cartesian coordinates are

$$
\left.\begin{array}{c}
\frac{\partial u}{\partial t}-f v+u \frac{\partial u}{\partial x}+v \frac{\partial u}{\partial y}=\frac{\tau_{x}}{h}-g^{\prime} \frac{\partial h}{\partial x}+\nu \nabla^{2} u, \\
\frac{\partial v}{\partial t}+f u+u \frac{\partial v}{\partial x}+v \frac{\partial v}{\partial y}=\frac{\tau_{y}}{h}-g^{\prime} \frac{\partial h}{\partial y}+\nu \nabla^{2} v, \\
\frac{\partial h}{\partial t}=-\frac{\partial h u}{\partial x}-\frac{\partial h v}{\partial y} .
\end{array}\right\}
$$

$h$ is the mixed-layer depth, $g^{\prime}=g \Delta \rho / \rho$ is the reduced gravity, with $\Delta \rho$ the density jump between the two layers. The internal Rossby radius of deformation $R=\left(g^{\prime} h_{0}\right)^{1 / 2} / f$ is a natural lengthscale of the problem. $\tau_{x}$ and $\tau_{y}$ are the components of the wind stress divided by density, expressed in units of $\mathrm{m}^{2} \mathrm{~s}^{-2}$. Laplacian friction with a dissipative coefficient $v$ has been introduced, following Gill (1984), to parameterize the vertical propagation of the inertial waves in the deeper layers that occurs in a continuously stratified ocean. Turbulent entrainment at the base of the mixed layer, that generates a damping effect roughly similar to the Laplacian friction, is not considered here for simplicity. Without loss of generality we assume that the wind blows in the $x$-direction, e.g. $\tau_{y}=0$.

Let us consider the response to a steady, spatially uniform wind switched on at $t=0$. When the advective and dissipative terms are neglected and $\tau_{x} / h$ is replaced by $\tau_{x} / h_{0}$ the linear response consists of a mean Ekman drift and free inertial oscillations. When the initial conditions vary in space, there are also free propagating inertial waves. Their frequency is given by the familiar dispersion relation:

$$
\omega^{2}=f^{2}+c^{2}\left(k^{2}+l^{2}\right)
$$


with $c=\left(g^{\prime} h_{0}\right)^{1 / 2}$. This solution is labelled as the zero-order solution.

\subsection{Linear theory}

A better knowledge of the dynamics of the wind-forced inertial motions can be gained through a detailed examination of the linearized equations obtained by retaining the dominant terms in (1). For that purpose, let us consider a dimensional analysis using a velocity scale, $U$, associated with the Ekman drift $\left(v_{s}=\tau_{x} / f h_{0}\right)$, the lengthscale, $L$, associated with the spatial heterogeneity of the waves and $f^{-1}$ as a timescale. We assume, first, that the Rossby number associated with the inertial waves, defined as $\epsilon=U / f L$, is small (i.e. $\epsilon \ll 1$ ). Secondly, the damping rate associated with the dissipative terms is supposed to be small (i.e. $v / f L^{2}=O(\epsilon)$ ). Then, using an expansion in $\epsilon$ for $h$, a dimensional analysis of the mixed-layer depth equation leads to the order $O\left(\epsilon^{2}\right)$ :

$$
h \approx h_{0}+h^{\prime}
$$

with $h^{\prime} / h_{0}=O(\epsilon)$. Using the same dimensional analysis for the momentum equations and keeping only the resonant terms (i.e. with the wave-wave interactions terms neglected), the resulting linearized equations are (when only terms of order $O(1)$ and $O(\epsilon)$ are retained):

$$
\left.\begin{array}{rl}
\frac{\partial u^{\prime}}{\partial t}-f v^{\prime}+v_{s} \frac{\partial u^{\prime}}{\partial y} & =-h^{\prime} \frac{\tau_{x}}{h_{0}^{2}}-g^{\prime} \frac{\partial h^{\prime}}{\partial x}+\nu \nabla^{2} u^{\prime}, \\
\frac{\partial v^{\prime}}{\partial t}+f u^{\prime}+v_{s} \frac{\partial v^{\prime}}{\partial y} & =-g^{\prime} \frac{\partial h^{\prime}}{\partial y}+\nu \nabla^{2} v^{\prime}, \\
\frac{\partial h^{\prime}}{\partial t} & =-h_{0}\left(\frac{\partial u^{\prime}}{\partial x}+\frac{\partial v^{\prime}}{\partial y}\right),
\end{array}\right\}
$$

where $u^{\prime}=u$ and $v^{\prime}=v-v_{s}$, with $v_{s}$ the Ekman drift velocity.

We look for solutions like $\exp (\mathrm{i}(k x+l y-\omega t))$. Then, using the non-dimensional variable: $X=\left(\omega-v_{s} l+\mathrm{i} v K^{2}\right) / f$ with $K^{2}=k^{2}+l^{2}$, we get the following dispersion relation:

$$
X^{3}+X^{2}\left(\frac{v_{s} l}{f}-\mathrm{i} \frac{\nu K^{2}}{f}\right)+X\left(1+R^{2} K^{2}-\mathrm{i} \frac{\tau_{x}}{f^{2} h_{0}} k\right)+2 \frac{v_{s} l}{f}-\mathrm{i} \frac{\nu K^{2}}{f}=0,
$$

with $R=\left(g^{\prime} h_{0}\right)^{1 / 2} / f$ the internal Rossby radius. From the dimensional analysis, the terms $v_{s} l / f, \nu K^{2} / f$ and $\left(\tau_{x} / f^{2} h_{0}\right) k$ are of order $O(\epsilon)$. So let us consider a solution like: $X=X_{0}+\epsilon X_{1}+O\left(\epsilon^{2}\right)$. From (4) we get:

$$
\frac{\omega}{f}=\left(1+R^{2} K^{2}\right)^{1 / 2}+\frac{v_{s} l}{2 f}\left(\frac{3+R^{2} K^{2}}{1+R^{2} K^{2}}\right)-\mathrm{i} \frac{\nu K^{2}}{2 f}\left(\frac{2+R^{2} K^{2}}{1+R^{2} K^{2}}\right)-\mathrm{i} \frac{\tau_{x} k}{f^{2} h_{0}\left(1+R^{2} K^{2}\right)^{1 / 2}} .
$$

The first two terms on the right-hand side of (5) represent respectively the classical frequency departure from $f$ owing to the isotropic dispersion, and the Doppler shift induced by the Ekman drift. The third (imaginary) term is the classical damping rate due to dissipation. The last term is new and can affect dramatically the dynamics of the system. It represents a growth or damping rate depending on the sign of $\tau_{x} k$. More precisely, amplitudes of waves that propagate against the wind $\left(\tau_{x} k<0\right)$ can grow exponentially. On the other hand, waves that propagate in the wind direction $\left(\tau_{x} k>\right.$ 0 ) should be damped. Waves propagating perpendicular to the wind are not affected by this term. This dispersion relation therefore reveals a forced instability of the inertial waves. Coming back to the linearized equations (3), a more precise examination shows 


\begin{tabular}{|c|c|c|c|c|c|c|c|c|}
\hline \multirow[b]{2}{*}{ Run } & \multirow[b]{2}{*}{ Forcing } & \multirow[b]{2}{*}{ Equations } & \multicolumn{4}{|c|}{ Parameters } & \multicolumn{2}{|c|}{ Non-dimensional } \\
\hline & & & $g^{\prime}$ & $h_{0}$ & $\tau$ & $\nu$ & $\tau^{*}$ & $v^{*}$ \\
\hline \multicolumn{9}{|c|}{ Dispersion of a patch } \\
\hline D1 & $T / h$ & Linearized & $2 \times 10^{-3}$ & 30 & $1.5 \times 10^{-4}$ & 10 & 0.2 & 0.017 \\
\hline D2 & $\tau / h_{\mathrm{g}}$ & Linearized & $2 \times 10^{-3}$ & 30 & $1.5 \times 10^{-4}$ & 10 & 0.2 & 0.017 \\
\hline D3 & $\tau / h$ & Nonlinear & $2 \times 10^{-3}$ & 30 & $1.5 \times 10^{-4}$ & 10 & 0.2 & 0.017 \\
\hline D4 & $\left(\tau+\tau^{\prime}\right) / h$ & Nonlinear & $2 \times 10^{-3}$ & 30 & $1.5 \times 10^{-4}$ & 10 & 0.2 & 0.017 \\
\hline \multicolumn{9}{|c|}{ Coastal cases } \\
\hline O1 & $\tau / h_{0}$ & Nonlinear & $2 \times 10^{-3}$ & 30 & $1.5 \times 10^{-4}$ & 50 & 0.2 & 0.083 \\
\hline $\mathrm{O} 2$ & $\tau / h$ & Nonlinear & $2 \times 10^{-3}$ & 30 & $1.5 \times 10^{-4}$ & 50 & 0.2 & 0.083 \\
\hline $\mathrm{O} 3$ & $r / h$ & Nonlinear & $1 \times 10^{-2}$ & 50 & $6 \times 10^{-4}$ & 20 & 0.17 & 0.004 \\
\hline \multicolumn{9}{|c|}{ Cyclonic (C) and anticyclonic (A) eddies } \\
\hline A0 & $\tau / h_{0}$ & Nonlinear & $2 \times 10^{-3}$ & 30 & $1.75 \times 10^{-4}$ & 50 & 0.24 & 0.083 \\
\hline A1 & $\tau / h$ & Nonlinear & $2 \times 10^{-3}$ & 30 & $1.75 \times 10^{-4}$ & 50 & 0.24 & 0.083 \\
\hline $\mathrm{A} 2$ & $\tau / h$ & Nonlinear & $2 \times 10^{-3}$ & 30 & $1.5 \times 10^{-4}$ & 50 & 0.2 & 0.083 \\
\hline A3 & $\tau / h$ & Nonlinear & $2 \times 10^{-3}$ & 30 & $1.75 \times 10^{-4}$ & 200 & 0.24 & 0.33 \\
\hline $\mathrm{CO}$ & $\tau / h_{0}$ & Nonlinear & $2 \times 10^{-3}$ & 30 & $1.75 \times 10^{-4}$ & 50 & 0.24 & 0.083 \\
\hline $\mathrm{Cl}$ & $T / h$ & Nonlinear & $2 \times 10^{-3}$ & 30 & $1.75 \times 10^{-4}$ & 50 & 0.24 & 0.083 \\
\hline $\mathrm{C} 2$ & $\tau / h$ & Nonlinear & $2 \times 10^{-3}$ & 30 & $1.5 \times 10^{-4}$ & 50 & 0.2 & 0.083 \\
\hline $\mathrm{C} 3$ & $\tau / h$ & Nonlinear & $2 \times 10^{-3}$ & 30 & $1.75 \times 10^{-4}$ & 200 & 0.24 & 0.33 \\
\hline
\end{tabular}

TABLE 1. List of experiments. Units are $\mathrm{m} \mathrm{s}^{-2}$ for the reduced gravity $g^{\prime}, \mathrm{m}^{2} \mathrm{~s}^{-2}$ for the wind stress $\tau$, $\mathrm{m}$ for $h_{0}$ and $\mathrm{m}^{2} \mathrm{~s}^{-1}$ for the Laplacian friction $\nu . \tau^{*}$ and $\nu^{*}$ are defined in the text. For all experiments, the Coriolis frequency is $f=10^{-4} \mathrm{~s}^{-1}$. See $\$ 3.1$ for the definition of variable wind stress $\tau^{\prime}$ in experiment D4.

that its origin is the forcing term $\tau_{x} / h$ present in the $u$-equation. In many studies $\tau_{x} / h$ is approximated as $\tau_{x} / h_{0}$ and therefore is considered as a constant forcing term. From our results, taking into account the $h$-variations in the forcing term triggers an instability mechanism of the inertial waves that, to our knowledge, has not been documented in the literature before KT93.

\subsection{Numerical results}

In order to confirm the existence of this forced instability mechanism and to better assess its effects, we have performed several numerical simulations using the linearized equations (3) as well as the fully nonlinear equations (1). The parameters of these experiments (labelled D1 to D3) are described in table 1.

Equations have been discretized using a standard finite-difference method on a staggered $\mathrm{C}$-grid in a doubly periodic domain. The situation we consider is the dispersion of a localized Gaussian kinetic energy perturbation, in the presence of a uniform steady wind stress. The value chosen for the stress $\left(\tau_{x}=1.5 \quad 10^{-4} \mathrm{~m}^{2} \mathrm{~s}^{-2}\right)$ corresponds to an eastward wind speed of about $10 \mathrm{~m} \mathrm{~s}^{-1}$. Initial conditions are:

$$
h_{0}=30 \mathrm{~m}, \quad v_{0}=0, \quad u_{0}=0.05 \exp \left(-\left(x^{2}+y^{2}\right) / \lambda^{2}\right) .
$$

The scale of the initial disturbance is $\lambda=12 \mathrm{~km}$, the size of the domain $400 \mathrm{~km}$, the boundary conditions are periodic. The Rossby radius wavelength is $2 \pi R=15.4 \mathrm{~km}$, and the ratio $\lambda / 2 \pi R$ is close to 1 . The fluid is almost inviscid: with $\nu=10 \mathrm{~m}^{2} \mathrm{~s}^{-1}$, the frictional timescale $\lambda^{2} / \nu$ is large (166 days) compared to the inertial period (17.5 h). All numerical integrations of (1) and (3) have been done with the same parameters and initial conditions. 


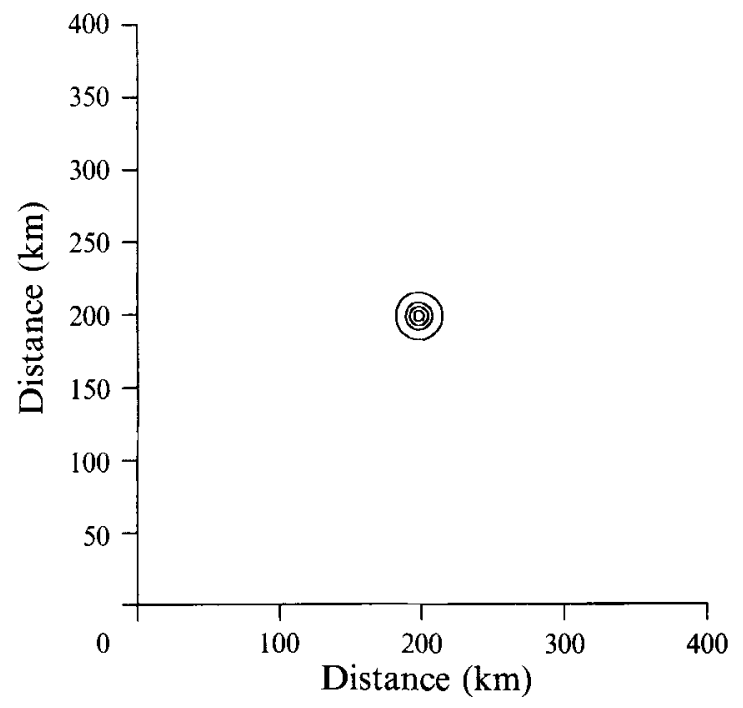

FIGURE 1. Kinetic energy integrated over the mixed-layer depth for the initial condition (15). Contour interval is $0.01 \mathrm{~m}^{3} \mathrm{~s}^{-2}$, lower contour is 0.001 .

Let us describe first the numerical solution D1 obtained with the linearized equations (3). Initially the kinetic energy perturbation is localized near the centre (figure 1). After one inertial period, there is a background energy related to the spatially uniform inertial oscillations. Its value, averaged over an inertial period and integrated over the mixed-layer depth is $\tau_{x}^{2} / f^{2} h_{0}=0.7510^{-1} \mathrm{~m}^{3} \mathrm{~s}^{-2}$. Later on the perturbation tends to spread isotropically. However, a patch of more energetic initial waves appears on the west side of the perturbation at $t=2$ inertial periods. The kinetic energy of these waves increases while they propagate in the southwest direction. In this patch, the local maximum of the kinetic energy (averaged over one inertial period) is larger than the background energy by a factor of 2 at $t=10$ and a factor of 3 at $t=15$ inertial periods (figures $2 a$ and $2 b$ ). The southward component of the patch propagation is simply due to the advection by the mean Ekman drift $v=-\tau_{x} / f h_{0}$. But the westward component of the patch propagation (against the wind) as well as the related kinetic energy growth are consequences of the instability mechanism described previously.

In order to confirm that the forcing term $\tau_{x} / h$ is indeed responsible for the energy growth, experiment D2 has been made with the same parameters and equations (3) but using $\tau_{x} / h_{0}$ as a forcing term instead of $\tau_{x} / h$. The inertial waves dynamics (figure 3 ) is strikingly different: the kinetic energy maximum at $t=15$ inertial periods is equal to the one at the first inertial period, i.e. very close to the background energy (note that the contour interval on figure 3 is 8 times smaller than on figure $2 b$ ). The perturbation has spread out in a circular pattern because of the radial wave dispersion. These results confirm that the forcing term $\tau_{x} / h$ is responsible for the energy growth.

With the parameter settings used, the theoretical growth rate (deduced from (5) and assuming that $l=0$ ) is equal to $\approx 0.06 f$. We have estimated a growth rate from the numerical results of the first experiment, using the time evolution of the amplitude of the mixed-layer depth variations as well as the one of the maximum velocity of the inertial waves. Amplitude of the mixed-layer depth variations is $3.5 \mathrm{~m}$ during the tenth inertial period and $10 \mathrm{~m}$ during the fifteenth inertial period. This leads to a growth rate of $0.033 f$. The velocity amplitude has a maximum value equal to $0.084 \mathrm{~m} \mathrm{~s}^{-1}$ at $t=10$ and $0.14 \mathrm{~m} \mathrm{~s}^{-1}$ at $t=15$ inertial periods, leading to a growth rate of $0.016 f$. These 

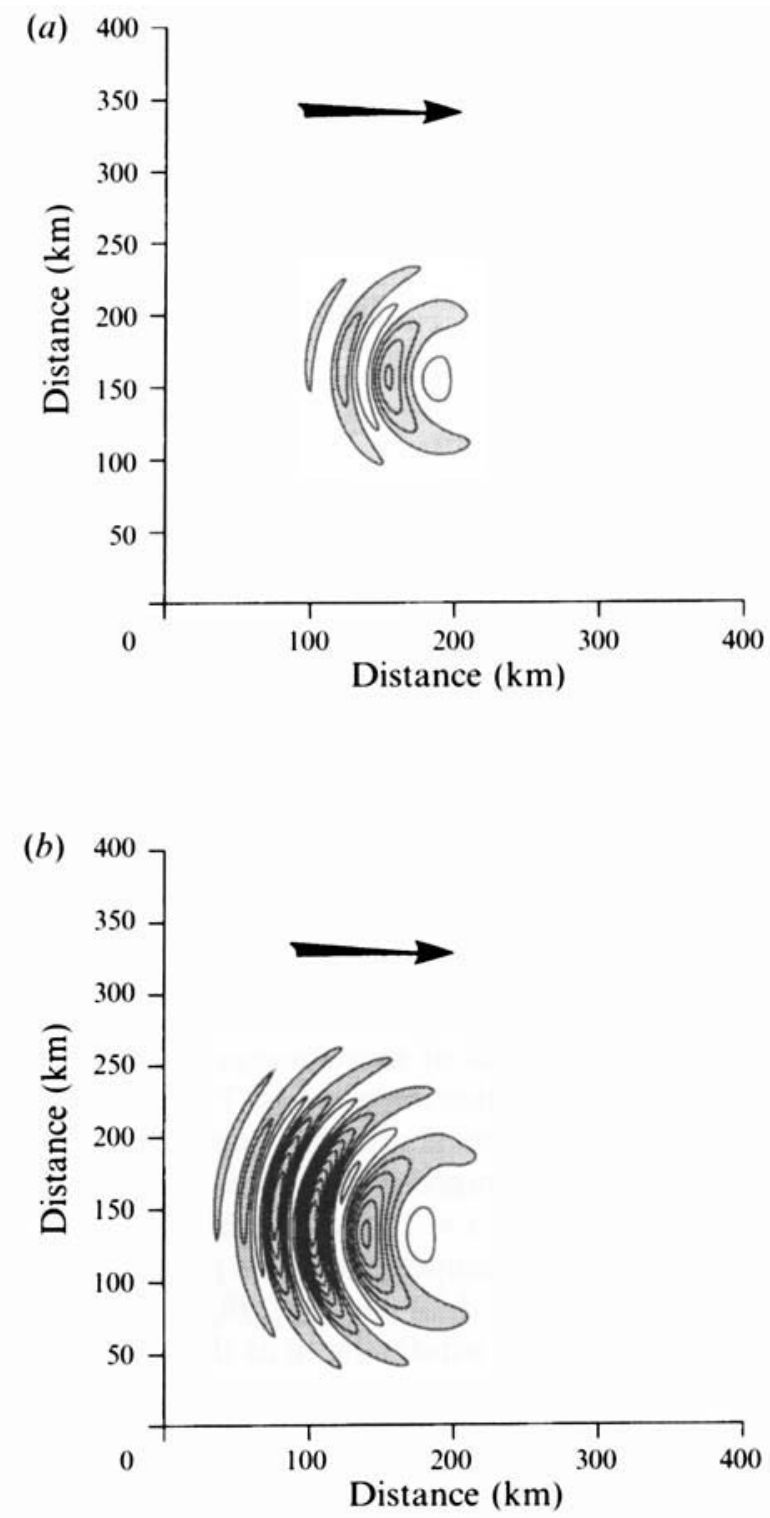

FIGURE 2. Kinetic energy (integrated over the mixed-layer depth and averaged one inertial period) for experiment Dl, (a) at $t=10$ inertial periods and $(b)$ at $t=15$ inertial periods. Contour interval is $0.02 \mathrm{~m}^{3} \mathrm{~s}^{-2}$. The shaded area shows energy levels higher than $0.08 \mathrm{~m}^{3} \mathrm{~s}^{-2}$. The wind direction is indicated by an arrow.

growth rates are significant but smaller than the theoretical one. One reason is that the theoretical one is calculated assuming the optimal situation: that is, the waves are assumed to be purely zonal and monochromatic. This is not true in the situation considered. Indeed, at least during the first inertial periods, the most unstable wave is not large enough to dominate the contribution of all other Fourier components to the amplitudes of $h$ and $u$.

A last numerical simulation (D3) using the fully nonlinear equations (1) has been performed in order to assess the effects of the nonlinear advective terms neglected in (3), and in particular the effects of the wave-wave interactions. The kinetic energy plot 


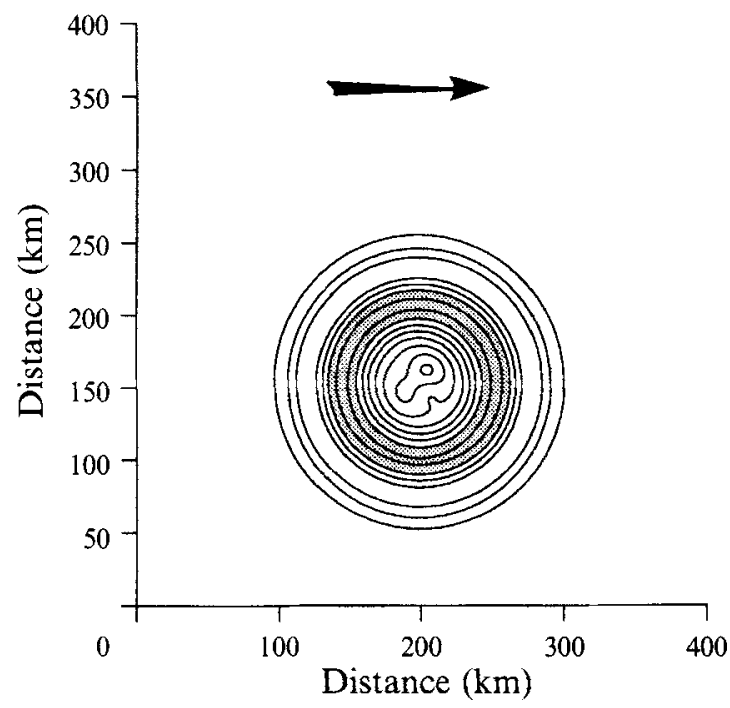

FiguRE 3. Kinetic energy (integrated over the mixed layer depth and averaged one inertial period) at $t=15$ inertial periods for experiment D2. Contour interval is $0.0025 \mathrm{~m}^{3} \mathrm{~s}^{-2}$. The shaded area shows energy levels higher than $0.08 \mathrm{~m}^{3} \mathrm{~s}^{-2}$. The wind direction is indicated by an arrow.

at $t=15$ (not shown) is almost identical to figure $2(b)$. This means that the linearized equations (3) capture most of the physics of the system: the wave--wave interactions do not affect significantly the instability mechanism.

\subsection{Physical explanation}

A physical understanding of the instability mechanism can be gained by studying the energy balance. We derive a domain-integrated energy equation from the linearized system (3) by multiplying the momentum equations by $h_{0} u^{\prime}$ and $h_{0} v^{\prime}$, respectively $\left(u^{\prime}, v^{\prime}\right.$ is the velocity with the mean Ekman drift substracted):

$$
\frac{\mathrm{d}}{\mathrm{d} t} \iint \frac{1}{2} h^{\prime}\left(u^{\prime 2}+v^{\prime 2}\right)+g^{\prime} h^{\prime 2}=-\frac{\tau_{x}}{h_{0}} \iint u^{\prime} h^{\prime}+v \iint h u \nabla^{2} u^{\prime}+h v \nabla^{2} v^{\prime}+(\ldots) .
$$

This equation shows that the energy growth (of order $\epsilon$, since $h^{\prime} / h_{0}=O(\epsilon)$ ) results from the correlation of $h^{\prime}$ and $u^{\prime}$. This was explained in KT93 and is illustrated by figure 4 . For classical linear inertial oscillations there is no growth because $h=h_{0}$ and the average of $u$ over one inertial period is zero. For inertial waves solution of (3), let us consider first waves with $k \tau_{x}<0$. The phase of $h^{\prime}$ and $u^{\prime}$ for those waves is such that $h^{\prime}$ is minimum when $u^{\prime}$ is in the same direction as the wind, and maximum when $u^{\prime}$ is against the wind, leading from (7) to an energy growth. For waves with $k \tau_{x}>0$ the situation is reversed and leads to a net energy loss.

In the nonlinear case, the total energy equation is obtained by multiplying the momentum equations by $h u$ and $h v$, respectively:

$$
\frac{\mathrm{d}}{\mathrm{d} t} \iint \frac{1}{2} h\left(u^{2}+v^{2}\right)+g^{\prime} h^{2}=\tau_{x} \iint u+v \iint h u \nabla^{2} u+h v \nabla^{2} v .
$$

In that case the energy growth is not simply related to the $\left(u^{\prime}, h^{\prime}\right)$ correlation but rather results from the existence of a time- and domain averaged velocity $\bar{u}$. Such a velocity averaged in time and space over the periodic domain cannot exist when the nonlinear 
(a)

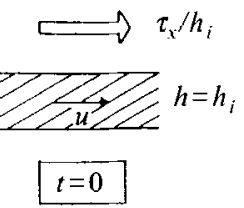

(b)

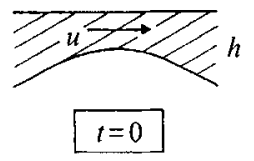

$(c)$

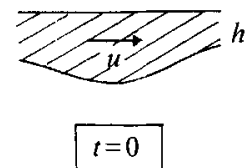

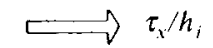

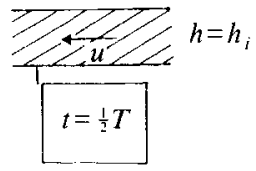

$\Longrightarrow \tau_{x} / h$
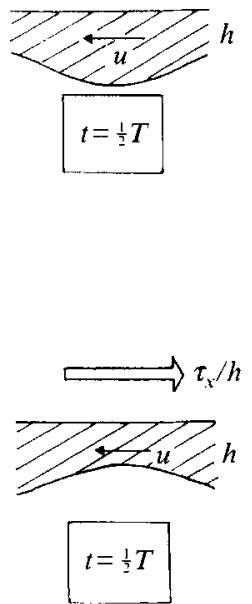

FIGURE 4. Sketch of the inertial resonance mechanism (reproduced from Klein \& Treguier 1993). $T$ represents an inertial period. (a) Linear case. (b) Nonlinear $k \tau_{x}<0$. (c) Nonlinear $k \tau_{x}>0$.

advective terms are discarded (this is readily shown by integrating the momentum equations over the domain). A time-mean zonal flow $\bar{u}$ in the direction of the wind stress is found indeed in nonlinear experiment D3, but it is negligible in linearized experiment D1. Even though it leads to a significant energy growth in D3, $\bar{u}$ is rather weak (the local maximum is $0.01 \mathrm{~m} \mathrm{~s}^{-1}$ during the 15 th inertial period). Moreover it is not uniform in space: the positive domain-average results from a complicated pattern of positive and negative values. In fact, (8) shows that small domain-averaged mean velocities can generate a large energy growth. For the parameters of our experiments, a doubling of the initial kinetic energy could be obtained in one inertial period with a domain-averaged zonal velocity of only $0.008 \mathrm{~m} \mathrm{~s}^{-1}$. The main symptom of the instability mechanism is therefore the energy growth of the oscillations, rather than a mean flow generation.

\subsection{Characteristics of the unstable waves in dissipative systems}

\subsubsection{Growth rate}

From (5) the growth of the wind-forced inertial waves triggered by the instability mechanism can be significantly affected, and even cancelled, by the dissipative mechanisms. Note again that the Laplacian friction present in (1) has been introduced to implicitly parameterize the vertical propagation of the inertial waves in the deeper layers that occur in a real stratified ocean. The competition between the instability mechanism (proportional to the horizontal wavenumber) and the dissipative 
mechanisms (proportional to the square of the horizontal wavenumber) leads to a lengthscale selection that is a function of the magnitude of the wind stress and of the dissipative coefficient $\nu$. In order to assess this scale selection, the effective growth rate displayed by (5) (i.e. the two imaginary terms) has been rewritten with the forcing and dissipative parameters $\tau_{x}$ and $\nu$ non-dimensionalized as: $\tau_{x}^{*}=\tau_{x} /\left(R h_{0} f^{2}\right)$, and $\nu^{*}=$ $v /\left(R^{2} f\right)$ with $R$ the Rossby radius defined as before. The wavenumbers are nondimensionalized as: $\left(k^{*}, l^{*}\right)=(k R, l R)$. The effective growth rate $\gamma / f$ is:

$$
\frac{\gamma}{f}=\frac{k^{*}}{2\left(1+K^{* 2}\right)^{1 / 2}}\left[-\tau_{x}^{*}-\frac{\nu^{*} K^{* 2}}{k^{*}} \frac{\left(2+K^{* 2}\right)}{\left(1+K^{* 2}\right)^{1 / 2}}\right]
$$

\subsubsection{Discussion}

The parameter settings used in this study involve $\tau_{x}^{*}$-values that range from 0.1 to 0.4 . These values can be attained with a wind stress varying between $10^{-4} \mathrm{~m}^{2} \mathrm{~s}^{-2}$ and $6 \times 10^{-4} \mathrm{~m}^{2} \mathrm{~s}^{-2}$ (wind speed of 10 to $25 \mathrm{~m} \mathrm{~s}^{-1}$ ), and with a shallow mixed layer of $30 \mathrm{~m}$ corresponding to a Rossby radius of deformation of between $2 \mathrm{~km}$ and $8 \mathrm{~km} . \nu^{*}$ is between 0.002 and 0.2 , if one assumes that the $\nu$-values can vary between $20 \mathrm{~m}^{2} \mathrm{~s}^{-1}$ (for a sharp and thin seasonal thermocline) and $200 \mathrm{~m}^{2} \mathrm{~s}^{-1}$ (for a thick seasonal thermocline) (see Gill 1984 and KT93). From (9), waves with a propagation parallel to the wind stress (i.e. with $l=0$ in the situation considered) are the ones for which the growth rate is the largest. So let us consider this situation and let us examine first the growth rates for different values of $\nu^{*}$ in the particular case $\tau^{*}=0.2$ (figure $5 \mathrm{a}$ ). From the marginal curve (corresponding to $\gamma / f=0$ ) the wavenumber $k^{*}$ of the unstable waves can vary between 0 and -1 when the dissipation is large (i.e. for $\tau^{*} / \nu^{*}<2$ ). The width of the unstable region increases when the dissipation decreases, and in the inviscid limit all negative wavenumbers are unstable. However, the wavenumber associated with the most unstable wave does not vary so much: it goes from $k^{*}=-0.5$ when $\tau^{*} / \nu^{*}=2$ to $k^{*}=-1.2$ when $\tau^{*} / \nu^{*}=10$. Therefore, for a large range of variation of $\tau^{*} / \nu^{*}$, the wavelength of the most unstable wave is of the order of the Rossby radius wavelength. We have checked through a detailed examination of (9) that these results hold for the $\tau^{*}$-values between 0.1 and 0.4 .

For a given value of $\tau^{*}$, the maximum growth rate strongly depends on the dissipation coefficient $\nu^{*}$. For example, with $\tau^{*}=0.2$ (figure $5 a$ ), it goes from 0.012 (when $\tau^{*} / \nu^{*}=1$ ) up to the upper limit $\tau^{*} / 2=0.1$ (when $\nu^{*}=0$ ). Figure $5(b)$ shows the contours of the maximum growth rate as a function of $\tau^{*}$ and $\nu^{*}$. If we choose (arbitrarily) $\gamma / f=0.02$ as a value large enough for the instability mechanism to produce significant effects, this figure reveals that the instability mechanism can affect the mixed-layer dynamics for a large range of $\tau^{*}$ and $\nu^{*}$-values. The only constraint is the wind stress duration. When $\gamma / f=0.02$ a wind stress duration of about 6 inertial periods is required for the mixed-layer amplitude to be multiplied by 2 . This duration is reduced to only 2 inertial periods when $\gamma / f=0.06$.

The order of magnitude of the maximum growth rate is well described by the empirical formula:

$$
\frac{\gamma_{m}}{f}=\frac{\tau^{*}}{2}\left(\frac{\tau^{*} / \nu^{*}}{8+\left(\tau^{*} / \nu^{*}\right)}\right)
$$

This expression has been established by matching in the simplest fashion the upper limit, $\frac{1}{2} \tau^{*}$, for very weakly dissipative systems and the lower limit, $\tau^{* 2} / 16 v^{*}$, for more dissipative systems. Let us stress that $(10)$ is empirical and gives only an upper bound of the real energy growth that will occur in realistic, nonlinear situations. Indeed the 

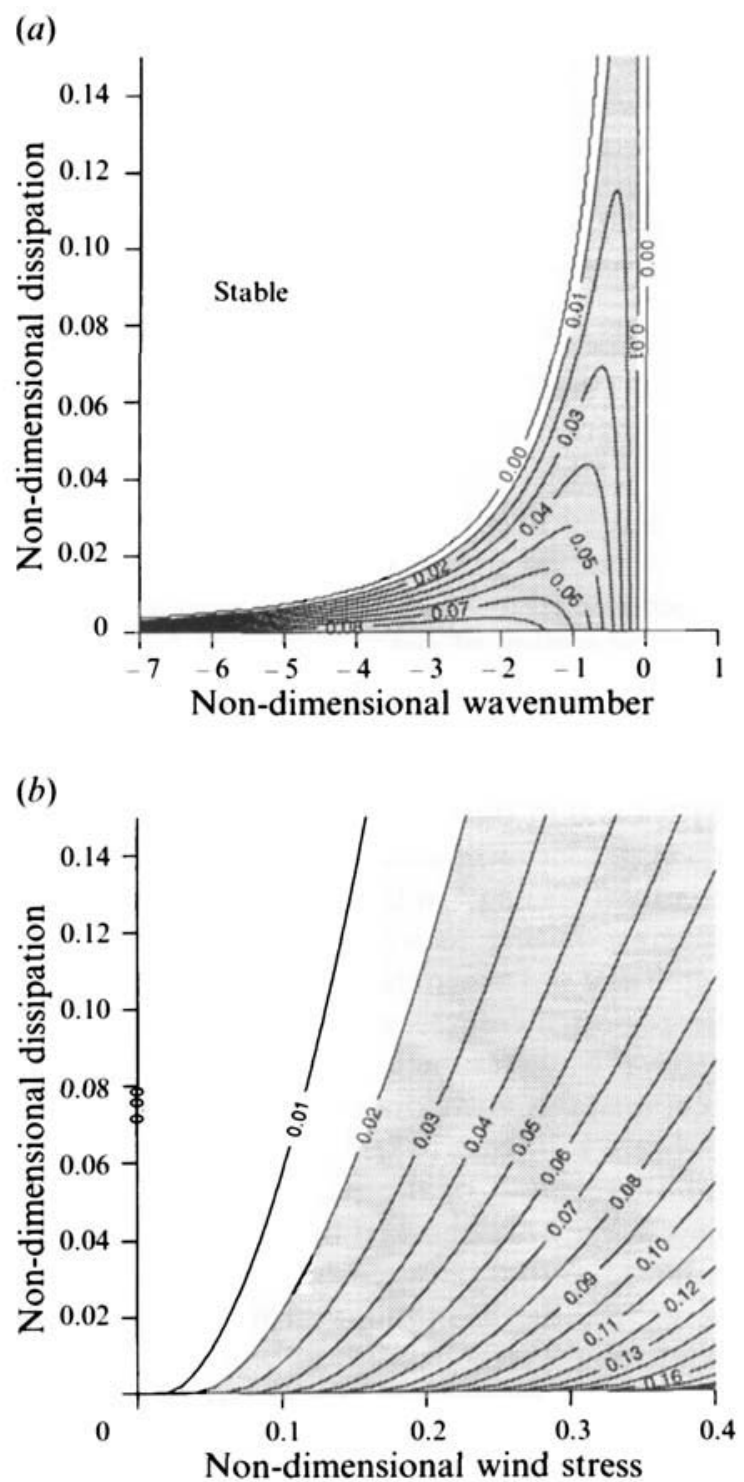

FIGURE 5. (a) Non-dimensional growth rate (from (9) with $l^{*}=0$ ) as a function of the wavenumber $k^{*}$ and the dissipation coefficient $\nu^{*}$ for $\tau^{*}=0.2$. The shaded area is the unstable region with growth rate larger than 0.01. (b) Maximum non-dimensional growth rate (from (9) with $l^{*}=0$ ) as a function of $\tau^{*}$ and $v^{*}$.

expression of this maximum growth rate that results from the linear theory assumes that the waves are monochromatic and that their wavenumber is parallel to the wind stress.

\section{Robustness of the instability mechanism in simple oceanic situations}

3.1. Effect of a time-varying wind stress

Consequences of the instability mechanism have been examined so far in the case of steady winds. It also happens with fluctuating winds, provided the time-mean is non- 


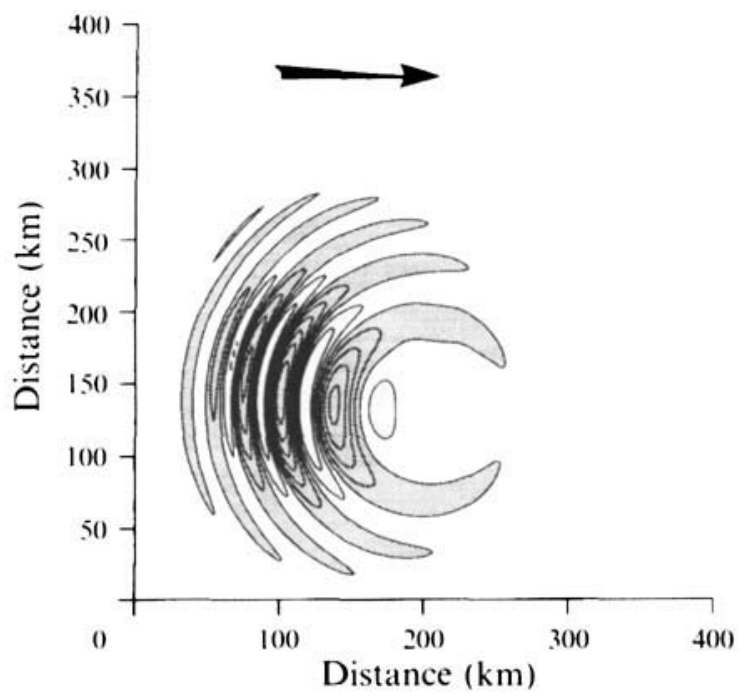

Figure 6. Kinetic energy (integrated over the mixed-layer depth and averaged one inertial period) at $t=15$ inertial periods for experiment D4. Contour interval is $0.05 \mathrm{~m}^{3} \mathrm{~s}^{-2}$. The shaded area shows energy levels higher than $0.25 \mathrm{~m}^{3} \mathrm{~s}^{-2}$. The wind direction is indicated by an arrow.

zero and of the same order as the fluctuations. This is demonstrated by repeating experiment D3 with a fluctuating wind stress (D4). A random wind stress series is generated, with the same steady component as the one considered before (i.e. $\overline{\tau_{x}}=$ $1.5 \times 10^{-4} \mathrm{~m}^{2} \mathrm{~s}^{-2}, \overline{\tau_{y}}=0$ ) and with variable components with r.m.s. amplitudes $A\left(\tau_{x}\right)$, $A\left(\tau_{y}\right)$ in both directions. Usually, the wind stress strength varies more than its direction. To model this effect we choose $A\left(\tau_{x}\right)=1.5 \times 10^{-4} \mathrm{~m}^{2} \mathrm{~s}^{-2}$, and a smaller value $A\left(\tau_{y}\right)=$ $0.5 \times 10^{-4} \mathrm{~m}^{2} \mathrm{~s}^{-2}$. The wind stress fluctuations in both directions are represented by Markovian processes of characteristic time $2 \mathrm{~h}$ as in Treguier \& Hua (1987). The numerical results reveal that the background energy averaged over an inertial period (related to the spatially uniform inertial oscillations) is no longer constant and has a maximum amplitude larger by a factor of 3 than the one related to the steady wind stress case. This is entirely due to the wind stress fluctuations (see Gill 1982). However, despite this difference, the response to this fluctuating wind stress in the nonlinear case appears to be qualitatively similar to the steady wind stress case: the initial perturbation starts to spread away in a circular pattern, but a patch of more energetic inertial waves appears on the west side of the perturbation after the first inertial period. The maximum of the kinetic energy (averaged over an inertial period) of this patch steadily increases while the related inertial waves propagate westward (figure 6). Quite remarkably, the time evolution of the maximum $h$ amplitude in experiment D4 is very close to experiment D1 (steady wind stress case), i.e. $3.6 \mathrm{~m}$ during the tenth inertial period and $11 \mathrm{~m}$ during the fifteenth. This represents an effective growth rate of $0.035 f$. The maximum velocity increases from $0.12 \mathrm{~m} \mathrm{~s}^{-1}$ to $0.22 \mathrm{~m} \mathrm{~s}^{-1}$ within five inertial periods, leading to an effective growth rate of $0.019 f$, again quite similar to the steady wind stress case.

So it appears that the instability mechanism is not sensitive to the wind stress fluctuations insofar as these fluctuations have a typical timescale smaller than the inertial period. Only the mean wind stress, i.e. the average over several inertial periods, is important for the instability mechanism to work. This feature has been checked with 
a numerical simulation similar to the preceding one but involving only the wind stress fluctuations (the mean value was set to zero). In that case the numerical results have not displayed any difference using $\tau_{x} / h$ or $\tau_{x} / h_{0}$ as a forcing term; which means the instability mechanism does not occur in the absence of a mean wind.

The effect of a wind varying both in space and time has also been investigated. Such a wind field can, by itself, generate instability since the forced response has a non-zero wavenumber $k$. However, the wind stress scales are rather large (more than $200 \mathrm{~km}$ ) and experiments using a realistic space-time wind spectrum as the only source of spatial variability show little difference between the $\tau_{x} / h$ and $\tau_{x} / h_{0}$ cases. The main effect of instability in those cases is qualitative rather than quantitative: a systematic propagation towards the mean wind and smaller spatial scales of the response are observed, but the energy growth in the upper layer remains unsignificant.

\subsection{Inertial waves generated at a horizontal boundary}

Wind-induced inertial waves generated at a horizontal boundary (such as a coast) have a wide spectrum of horizontal scales, and therefore some of them can be affected by the instability mechanism.

One classical example of such a situation is the response of a coastal ocean to a sudden impulsive cross-shore wind stress. This situation is usually idealized through a $1 \frac{1}{2}$-layer ocean bounded by an infinite coast coincident with the $y$-axis. An offshore wind perpendicular to the coast suddenly arises at $t=0$. The ocean response is characterized by a wind set-up and an associated 'pulse' of internal waves with a wide wavenumber spectrum. The wind set-up consists of piling up water in the top layer against the leeward coast, with a spatial scale equal to the Rossby radius of deformation, $R$, defined in the preceding section. Later on the wind set-up remains with a distance of order $O(R)$ from the coast, but the pulse of inertial waves propagates with the shortest waves propagating the fastest (Millot \& Crepon 1981).

This situation was fully investigated by Crepon (1969) using a linear model (see also Csanady 1982). From the linear equations, a time-independent solution, which characterizes the wind set-up, is:

$$
h(x)=\frac{\tau_{x}}{f\left(g^{\prime} h_{0}\right)^{1 / 2}} \exp (-x / R)+h_{0} .
$$

Note that (11) holds for both offshore and onshore winds. The time dependent part of the linear solution includes wind set-up variations and, mainly, inertial oscillations of slowly decaying amplitude. The decay comes about through a dispersal of waves to infinity. Arhan (1973) used a nonlinear model to examine the same problem. He found the decaying response for the case of a wind blowing away from the shore. However, for a wind blowing towards the shore, his numerical results display a propagating front of internal waves with amplitude strongly growing rather than decaying.

We have calculated similar solutions using the equations (1) in the one-dimensional version (i.e. with $\partial / \partial y=0$ ). Two solutions have been calculated: one $(\mathrm{O} 1)$ with the forcing term approximated as $\tau_{x} / h_{0}$ and the other one (O2) with the full forcing term $\tau_{x} / h$. The parameters correspond to $\tau_{x}^{*}=0.2, \nu^{*}=0.08$ and to a Rossby radius of deformation $R \approx 2.45 \mathrm{~km}$ (table 1 ).

When the forcing term is approximated as $\tau_{x} / h_{0}$, inertial oscillations with decaying amplitude propagate offshore (figure $7 a$ ). This solution is very close to the one found by Crepon (1969). The wind set-up attains $7 \mathrm{~m}$ near the coast, but there is no significant 

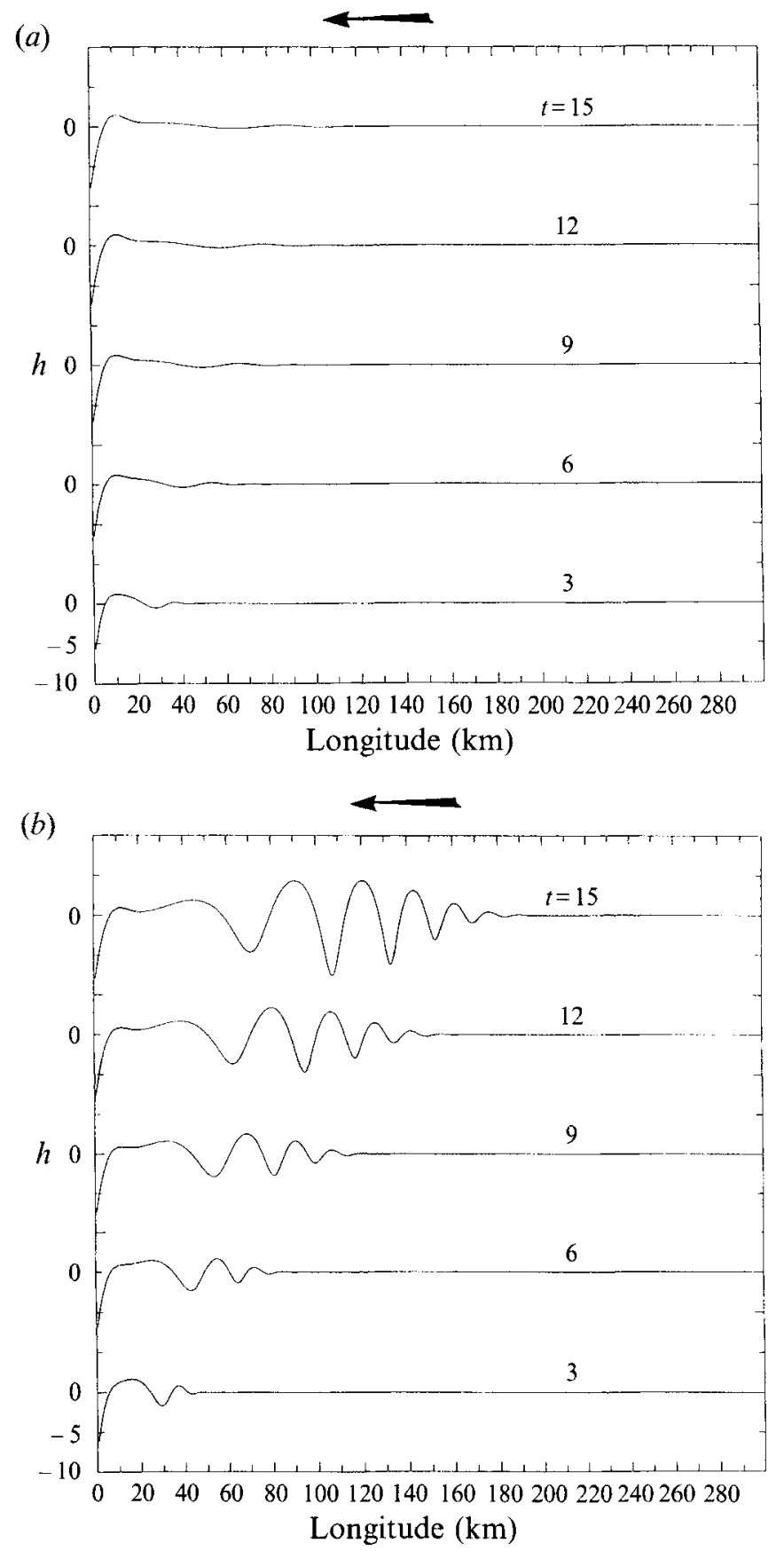

Figure 7. Mixed-layer depth perturbation $h-h_{0}$ as a function of distance from the coast at different times for $(a)$ experiment $\mathrm{O} 1$ and $(b)$ experiment $\mathrm{O} 2$. The wind direction is indicated by an arrow.

mixed-layer depth variation at a distance larger than $30 \mathrm{~km}$ from the coast. The kinetic energy does not increase. With the full forcing term $\tau_{x} / h$ taken into account the wind set-up near the near the coast is similar but there is a conspicuous 'exponential-like' growth of the inertial waves amplitude while they propagate away from the coast: the 
mixed-layer depth variation displays a sequence of upwellings and downwellings with an amplitude larger than $10 \mathrm{~m}$ at a distance of $100 \mathrm{~km}$ at 15 inertial periods (figure $7 \mathrm{~b}$ ). Note that, at this time, the wavelength corresponding to the largest amplitude is about $30 \mathrm{~km}$. The energy growth of the 'pulse' of inertial waves propagating off the coast clearly results from the instability mechanism.

Referring to the linear theory and to figure $5(b)$, the most unstable waves should have a theoretical effective growth rate $\gamma \approx 0.026 f$ and a wavenumber $k^{*} \approx 0.53$ corresponding to a wavelength equal to $30 \mathrm{~km}$, which compares well with the numerical results (figure $7 b$ ). The mixed-layer depth maximum amplitude (i.e. amplitude of the downwellings) varies from $3.5 \mathrm{~m}$ during the tenth inertial period to $7.6 \mathrm{~m}$ during the fifteenth inertial period. This leads to a growth rate of $\approx 0.024 f$, close to the theoretical one in this one-dimensional case. One feature, not observed in the preceding experiments, is the fact that upwellings are smaller than downwellings (figure $7 b$ ) ; hence a smaller growth rate for their amplitude. We have checked numerically that this is due to the asymmetric effect of the nonlinear advective terms on the upwellings and downwellings. These nonlinear advective terms are much efficient in this coastal situation (compared with the experiments of \$2.2) because the mixed-layer depth varies more relative to its initial value. The maximum amplitude of the velocity associated with the inertial waves varies between $0.11 \mathrm{~m} \mathrm{~s}^{-1}$ during the tenth inertial period to $0.16 \mathrm{~m} \mathrm{~s}^{-1}$ during the fifteenth inertial period; hence an effective growth rate of $\approx 0.012 f$, smaller than the theoretical one. In addition to the arguments invoked before, the effects of the nonlinear advective terms explain these smaller growth rates.

Another case (O3), more appropriate to the coastal ocean and close to the one considered by Arhan (1973), has been simulated with $\tau_{x}^{*} \approx 0.17, \nu^{*} \approx 0.004$ (i.e. $\tau_{x}^{*} / \nu^{*}=40$ ) and $R \approx 7 \mathrm{~km}$. Note that the amplitude of the wind set-up is equal to the preceding one (i.e. $\approx 7 \mathrm{~m}$ ). With the full forcing term $\tau_{x} / h$ taken into account, there is again an 'exponential-like' growth of the inertial waves (figure 8). This growth is much larger than in the previous case: mixed-layer depth variations attain $16 \mathrm{~m}$ after 5 inertial periods at a distance of $180 \mathrm{~km}$ from the coast. The wavelength of the largest wave appears to be between $20 \mathrm{~km}$ and $30 \mathrm{~km}$, not far from the theoretical value of $25 \mathrm{~km}$. From the linear theory, the effective growth rate with this parameter settings is $\gamma \approx 0.064 f$. This agrees with the growth of the maximum downwelling amplitude $(0.062 f)$, while the growth of upwellings and the maximum velocity is again smaller.

Finally, similar simulations with the full forcing term $\tau_{x} / h$ taken into account but with the wind blowing offshore have been performed. In that case (not shown), the internal waves propagating offshore have a negative growth rate since $k \tau_{x}>0$ and they are very quickly damped.

These results reveal that the instability mechanism affects significantly the coastal oceanic response to a wind stress. Besides the favourable parameter settings, one reason is the rapid appearance (within one inertial period) of a 'pulse' of inertial waves with a wide wavenumber spectrum because of the presence of the coast. Hence the existence of an initial spatial variability of the inertial oscillations. However, a wind stress duration of at least several inertial periods is required to observe a significant growth of the inertial waves. The linearized instability theory appears to give useful information about the characteristics of the unstable waves in particular about their wavelength and the maximum amplitude of the mixed depth variations. The nonnegligible effects of the nonlinear advective terms explain that the effective growth rate of the r.m.s. velocity is not so large as the one given by the linear theory. 


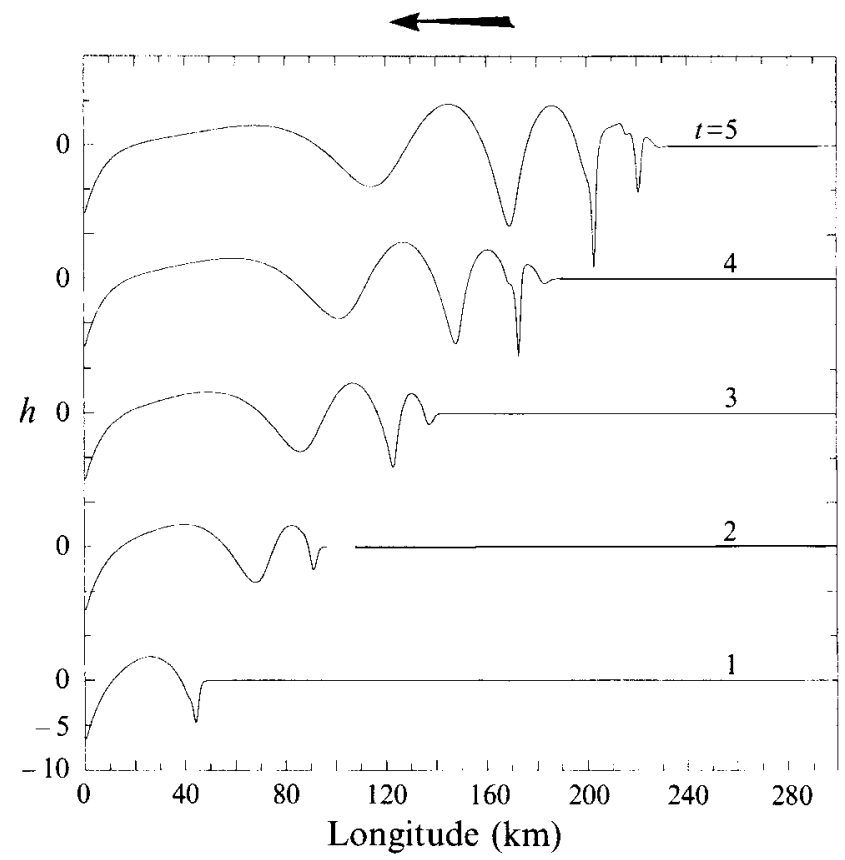

FIGURE 8. Mixed-layer depth perturbation $h-h_{0}$ as a function of distance from the coast at different times for experiment $\mathrm{O} 3$.

\section{The instability in presence of geostrophic eddies}

Oceanic jets and eddies are characterized by spatial scales from 20 to $200 \mathrm{~km}$ and timescales of a few months. Those mesoscale structures induce a significant spatial variability of the inertial waves (Kunze 1985). Therefore, they can trigger the windforced instability described in this paper. This was first demonstrated by KT93 for the case of a geostrophic jet uniform in the downstream direction. Here we extend those results to the case of a two-dimensional geostrophic eddy.

The equations we consider are based on (1). We assume that a geostrophic, nondivergent current $U, V$ exists in the mixed layer and below, and that this current is steady in time. The equations for the inertial velocities $u, v$ must take into account the nonlinear interactions with the geostrophic flow:

$$
\begin{aligned}
\frac{\partial u}{\partial t}-\left(f-\frac{\partial U}{\partial y}\right) v+u \frac{\partial U}{\partial x}+(u+U) \frac{\partial u}{\partial x}+(v+V) \frac{\partial u}{\partial y} & =\frac{\tau_{x}}{h}-g \frac{\Delta \rho}{\rho} \frac{\partial h}{\partial x}+\nu \nabla^{2} u \\
\frac{\partial v}{\partial t}+\left(f+\frac{\partial V}{\partial x}\right) u+v \frac{\partial V}{\partial y}+(u+U) \frac{\partial v}{\partial x}+(v+V) \frac{\partial v}{\partial y} & =\frac{\tau_{y}}{h}-g \frac{\Delta \rho}{\rho} \frac{\partial h}{\partial y}+\nu \nabla^{2} v \\
\frac{\partial h}{\partial t}+\frac{\partial h(u+U)}{\partial x}+\frac{\partial h(v+V)}{\partial y} & =0 .
\end{aligned}
$$

The geostrophic flow considered is a Gaussian eddy, defined by

$$
\psi=\psi_{0} \exp \left(-\left(x^{2}+y^{2}\right) / \lambda^{2}\right), \quad U=-\frac{\partial \psi}{\partial y}, \quad V=\frac{\partial \psi}{\partial x} .
$$

With $\lambda=32 \mathrm{~km}$, and $\psi_{0}$ such that the maximum velocity is $V_{M}=0.2 \mathrm{~m} \mathrm{~s}^{-1}$. Figure 9 


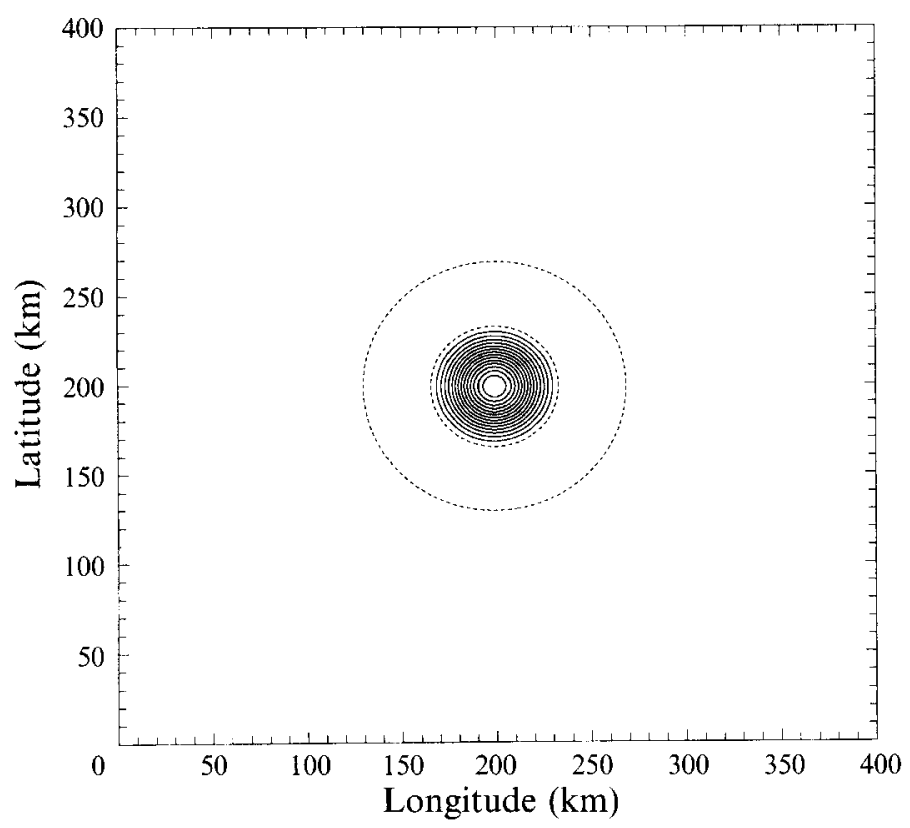

FIGURE 9. Non-dimensional vorticity $\zeta / f$ for a circular Gaussian cyclonic eddy. Contour interval is 0.02 , the dashed lines is the negative contour -0.01 .

represents the non-dimensional vorticity field in a cyclonic eddy. Note that the eddy core is surrounded by an annulus of vorticity of the opposite sign.

The strain and vorticity components of the geostrophic flow produce two important effects on the wind-driven inertial oscillations (Klein \& Hua 1988). First, they affect their amplitudes and in particular the mean Ekman drift whose components are:

$$
h v \approx-\frac{\tau_{x}}{f-\partial U / \partial y}, \quad h u \approx \frac{\tau_{x}}{f^{2}} \frac{\partial V}{\partial y} .
$$

As a consequence the mixed-layer depth can be significantly affected because of the non-zero Ekman divergence. On the other hand, the vorticity components modify the frequency of the inertial motions. This effect occurs through an effective Coriolis frequency, $f_{e}$, that replaces the Coriolis frequency in the dispersion relation:

$$
f_{e}(x)=(f(f+\zeta(x)))^{1 / 2},
$$

with $\zeta=\partial V / \partial x-\partial U / \partial y$ the vorticity of the geostrophic flow. Since the phase of the inertial oscillations $\omega t$ depends on $x$ through $f_{e}$, one consequence is an increasing spatial variability of the inertial motions that can be understood using a local expansion of the phase (Kunze 1985):

$$
\left.\begin{array}{rl}
\omega(x) t & \approx \omega(0) t+\frac{\partial f_{e}}{\partial x} x t+\frac{\partial f_{e}}{\partial y} y t+\ldots, \\
& \approx \omega(0) t-k x-l y,
\end{array}\right\}
$$

where $\{k, l\}=\left\{-t\left(\partial f_{e} / \partial x\right),-t\left(\partial f_{e} / \partial y\right)\right\}$ can be considered as the time-varying wavenumber that characterizes the spatial variability of the inertial waves.

In this situation, inertial waves can be affected by eddy dynamics as well as the forced instability mechanism. Experiments $\mathrm{AO}$ and $\mathrm{CO}$ (table 1) were performed using 
equations (12)-(14) with the forcing term approximated as $\tau_{x} / h_{0}$ in order to isolate the former effect.

\subsection{Effects of the Ekman drift divergence}

In the presence of a geostrophic flow, the divergence of the mean Ekman drift may induce systematic upwellings and downwellings and therefore modify the mixed-layer depth significantly. Such phenomena were first discussed by Niiler (1969) for a geostrophic jet. For a geostrophic eddy, such as the ones considered here, the divergence of the mean Ekman drift is

$$
\frac{\partial h u}{\partial x}+\frac{\partial h v}{\partial y} \approx \frac{\tau_{x}}{f^{2}} \frac{\partial \zeta}{\partial y}
$$

Hence, with eastward wind, the core of a cyclonic eddy displays a large upwelling in its southern part and a large downwelling in its northern part as shown in figure $10(a)$ (experiment $\mathrm{C} 0$ ). Note that the ring of negative vorticity around the cyclonic eddy is associated with a smaller upwelling (downwelling) farther north (south). The situation would be reversed in an anticyclonic eddy. The initial rate of upwelling or downwelling for the Gaussian eddy is estimated as:

$$
\frac{\partial h}{\partial t} \approx 6 \frac{V_{M}}{\lambda^{2}} \frac{\tau_{x}}{f^{2}}=1.8 \mathrm{~m} / \mathrm{day} .
$$

Indeed numerical results show that a depth close to $1.3 \mathrm{~m}$ is reached at the end of the first inertial period (figure $10 a$ ).

In the case of a rectilinear jet (for instance, $U=0$ and $\partial / \partial y=0$ ), those upwellings and downwellings persist at a constant rate when the wind is steady. However, in the case of an eddy, there are additional terms $U \partial h / \partial x$ and $V \partial h / \partial y$ in the mass equation (14). After a time comparable to the eddy timescale $\lambda / V_{M} \approx 2$ days, we find that the upwellings and downwellings are inhibited by the advective effects. Using (18) with the eddy advective terms taken into account, the estimated maximum value attained for the depth variations is therefore of the order:

$$
\frac{6}{\lambda} \frac{\tau_{x}}{f^{2}}=3.3 \mathrm{~m} .
$$

This shows that a steady wind stress over geostrophic eddies does not produce systematic upwelling and downwelling effects, contrary to the case of a geostrophic jet as examined by Niiler (1969). After the first inertial periods, the pattern of $h$ becomes very complicated and small-scale features appear, but the amplitude does not grow (figure $10 \mathrm{~b}$ ).

\subsection{Effects of the eddies on the inertial wave dynamics}

Let us now examine the specific effects of the eddy dynamics on the evolution of the inertial oscillations as revealed by the numerical experiments $\mathrm{C} 0$ and $\mathrm{A} 0$.

Inertial oscillations are established in the upper layer from the first inertial period. Let us stress again that the eddy vorticity affects their amplitude in the same way as for the mean Ekman drift. The r.m.s. velocity, roughly proportional to $\tau_{x} /\left[h_{0}(f-\partial U / \partial y)\right]$, is minimum in the core of a cyclonic eddy with two maxima north and south (figure $11 a$ ), whereas the situation is reversed in an anticyclonic eddy. Furthermore, the eddy vorticity shifts the local frequency 'seen' by the inertial oscillations. As noted before, this leads to a growing spatial variability with a time-dependent 'wavenumber' proportional to the vorticity gradient (17). Expression of the resulting group velocity (proportional to this wavenumber) shows that the energy should concentrate in 

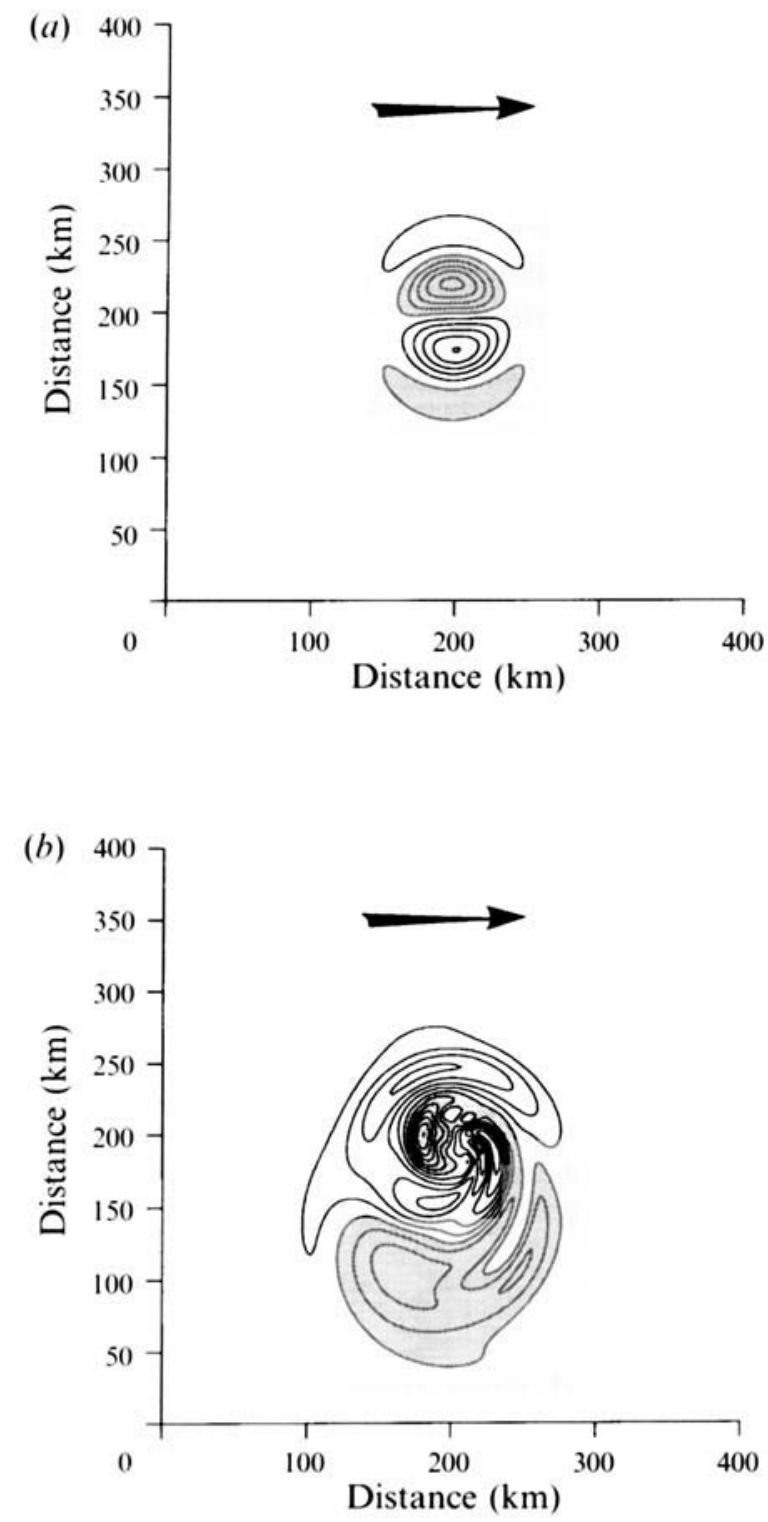

Figure 10. Mixed-layer depth perturbation $h-h_{0}$ for experiment $\mathrm{C} 0,(a)$ at the end of the first inertial period. Contour interval is $0.3 \mathrm{~m}$ with minimum contour $-1.35 \mathrm{~m} ;(b)$ at the end of the 20 th inertial period. Contour interval is $0.5 \mathrm{~m}$ with minimum contour $-2.25 \mathrm{~m}$. The shaded area indicates downwelling. The wind direction is indicated by an arrow.

negative vorticity regions, for example in the core of the anticyclonic eddy. On the other hand, the energy flux should be directed outwards in a cyclonic vorticity region (see Kunze 1985 for more details).

Let us examine first the cyclonic eddy case (experiment $\mathrm{C} 0$ ). As expected an energy minimum appears within the eddy core during the first inertial period (figure $11 \mathrm{a}$ ). It is associated with two energy maxima located north and south within the negative vorticity ring surrounding the core. Later on, the kinetic energy within the eddy core decreases (up to $50 \%$ within 20 inertial periods) because the vorticity is positive there, leading to the expulsion of the inertial oscillations. When the nonlinear terms involving 

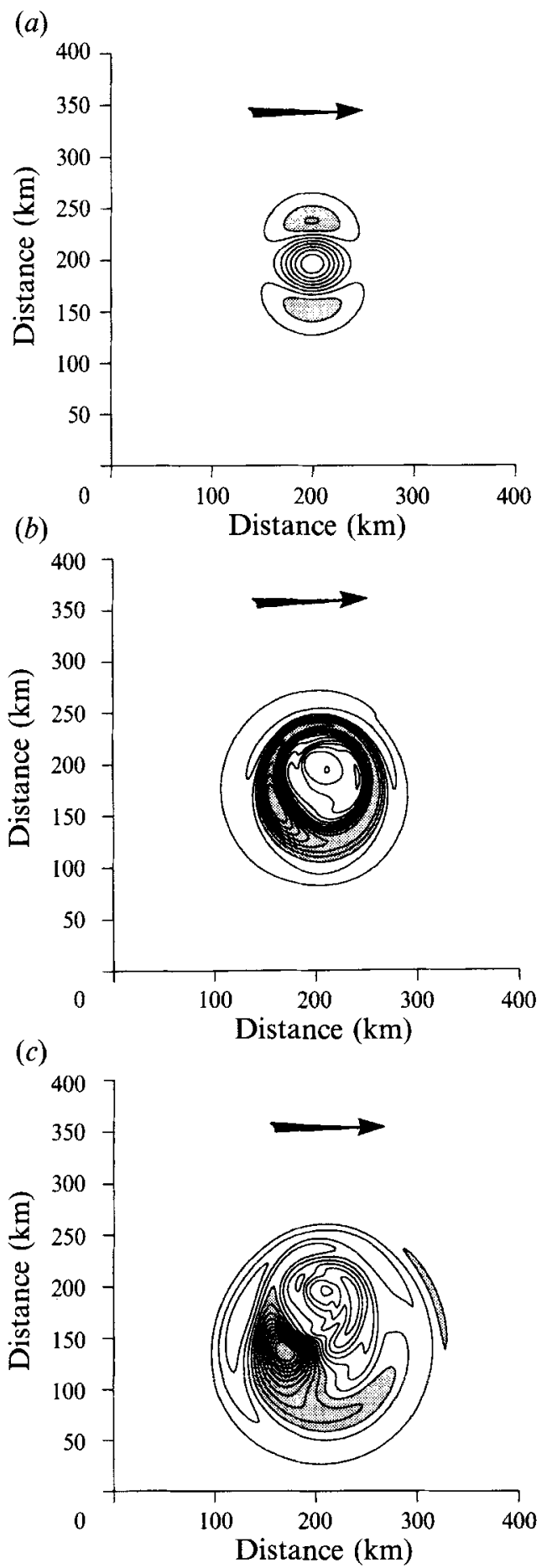

FIGURE 11. Experiment C0: kinetic energy (integrated over the mixed-layer depth and averaged one inertial period). The shaded area indicates energy higher than the background $\left(>0.11 \mathrm{~m}^{3} \mathrm{~s}^{-2}\right)$. (a) At $t=1$ inertial periods, contour interval is $0.005 \mathrm{~m}^{3} \mathrm{~s}^{-2} ;(b)$ at $t=10$ inertial periods, contour interval is $0.01 \mathrm{~m}^{3} \mathrm{~s}^{-2} ;(c)$ at $t=20$ inertial periods, contour interval is $0.01 \mathrm{~m}^{3} \mathrm{~s}^{-2}$. 

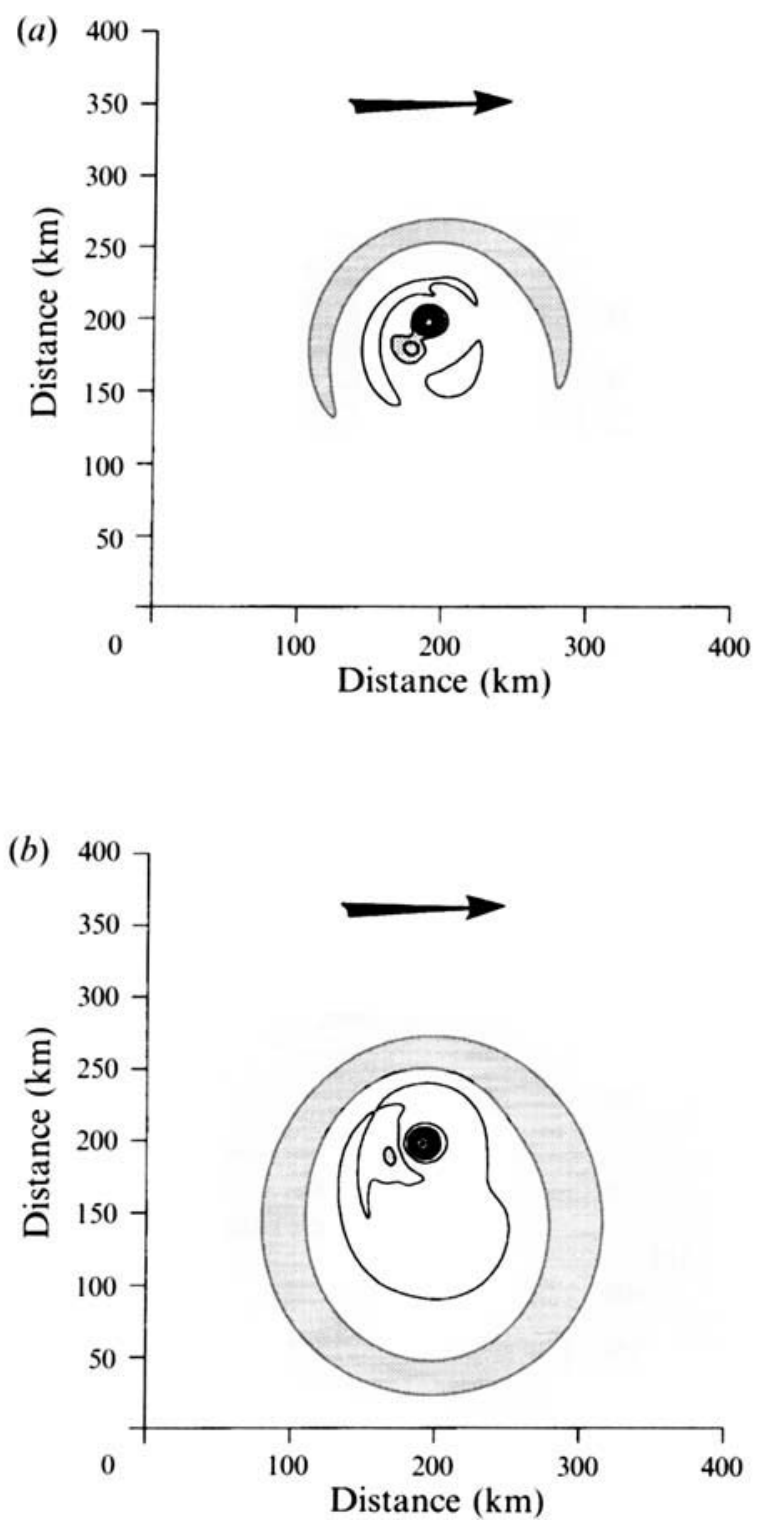

FIGURE 12. Experiment A0: kinetic energy (integrated over the mixed-layer depth and averaged one inertial period). The shaded area indicates energy higher than the background $\left(>0.11 \mathrm{~m}^{3} \mathrm{~s}^{-2}\right)$. Contour interval is $0.03 \mathrm{~m}^{3} \mathrm{~s}^{-2}$. (a) At $t=10$ inertial periods; (b) at $t=20$ inertial periods.

products of $u$ and $v$ (wave-wave interactions) are discarded, the two energy maxima spread around the core in a circular pattern. In the fully nonlinear case their evolution is more complicated. The energy maximum located initially on the south edge of the eddy ring quickly disappears. The one on the north side increases (up to twice its initial value after 20 inertial periods), rotates around the eddy, and finally remains locked on the southwest side (figures $11 b$ and $11 c$ ) while its amplitude slightly increases (see curve $\mathrm{C} 0$ in figure 13). The energy appears to be trapped within this maximum, whose exact location depends on the magnitude of the eddy velocity and of the radial propagation induced by the eddy vorticity as well as on the Ekman drift: for example, when the 


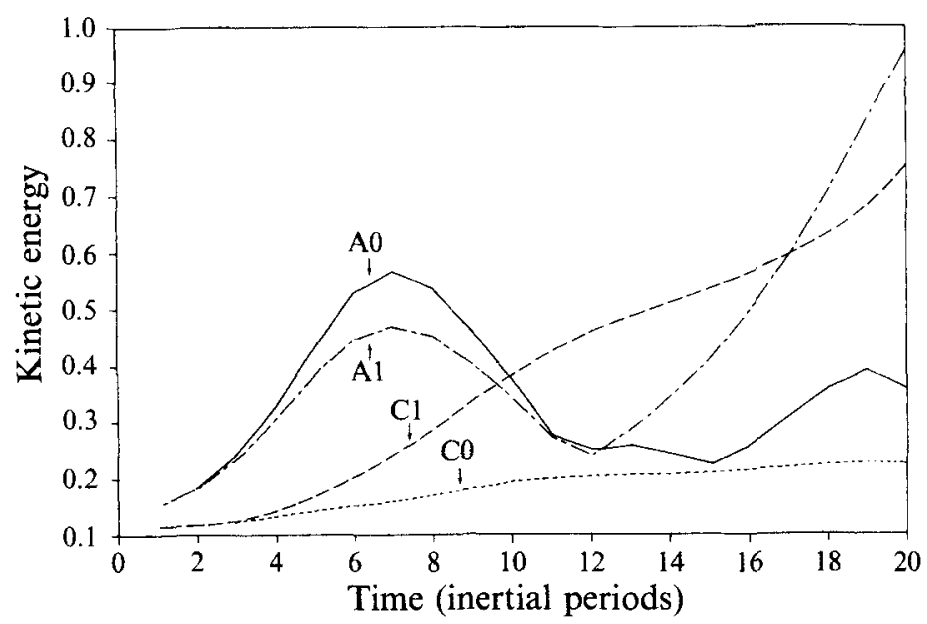

FlgURE 13. Time series of the maximum kinetic energy (integrated over the mixed-layer depth and averaged over successive inertial periods, in $\mathrm{m}^{3} \mathrm{~s}^{-2}$ ) for experiments $\mathrm{A} 0, \mathrm{Al}, \mathrm{C} 0$, and $\mathrm{Cl}$.

eddy velocity and vorticity are twice as big, this maximum is locked on the south edge of the eddy. Finally, note that in experiment $\mathrm{C} 0$ the maximum amplitude of the mixedlayer depth variation is $5 \mathrm{~m}$ (figure $10 b$ ).

Numerical results with an anticyclonic eddy (experiment A0), when compared with the preceding case, display an antisymmetric kinetic energy distribution during the first inertial period: there is an energy maximum in the negative vorticity core region and two minima north and south of the positive vorticity ring surrounding the eddy core. Later on the two minima disappear but after 6 inertial periods some inertial waves have escaped from the ring and propagate radially outside the eddy (figure 12a). Inside the negative vorticity core the kinetic energy maximum oscillates (figure 13, curve A0). This time evolution can be explained from the propagation of the inertial waves: because of the radial symmetry, these waves first propagate towards the centre and then propagate towards the edges after some time $T$. On the analogy of the propagation of a circular wavefront, this time $T$ can be roughly estimated as the ratio of the eddy radius to the group velocity. Later on the energy is reflected off the edge of the eddy and starts to propagate again towards the centre. Such reflections at the boundary of the negative vorticity region are discussed by Kunze (1985). Therefore the energy maximum within the eddy core behaves as a damped oscillator. Figure $12(b)$ shows the kinetic energy during the 20th inertial period. The concentration of the energy maximum is made clear by a comparison with the core shape during the first inertial period (which is antisymmetric to figure 11 a). Note also that the inertial energy that has escaped from the eddy is larger at that time than in the cyclonic case. The southward shift of the energy distribution is due to the Ekman drift.

\subsection{Effects of the forced instability mechanism}

Two experiments $\mathrm{A} 1$ and $\mathrm{Cl}$, similar to $\mathrm{A} 0$ and $\mathrm{C} 0$ but with $\tau_{x} / h$ as the forcing term in (12), have been performed in order to assess the effects of the instability mechanism.

Let us examine first the situation with an anticyclonic eddy, experiment A1. The kinetic energy distribution maps of A1 display the same time evolution as A0 during the first 5 inertial periods. At $t=10$ inertial periods a patch of inertial waves has escaped outside the eddy, but only the ones propagating upwind (westward) have survived (figure $14 a$ ). Their kinetic energy has a maximum value of $\approx 0.13 \mathrm{~m}^{3} \mathrm{~s}^{-2}$ at that 

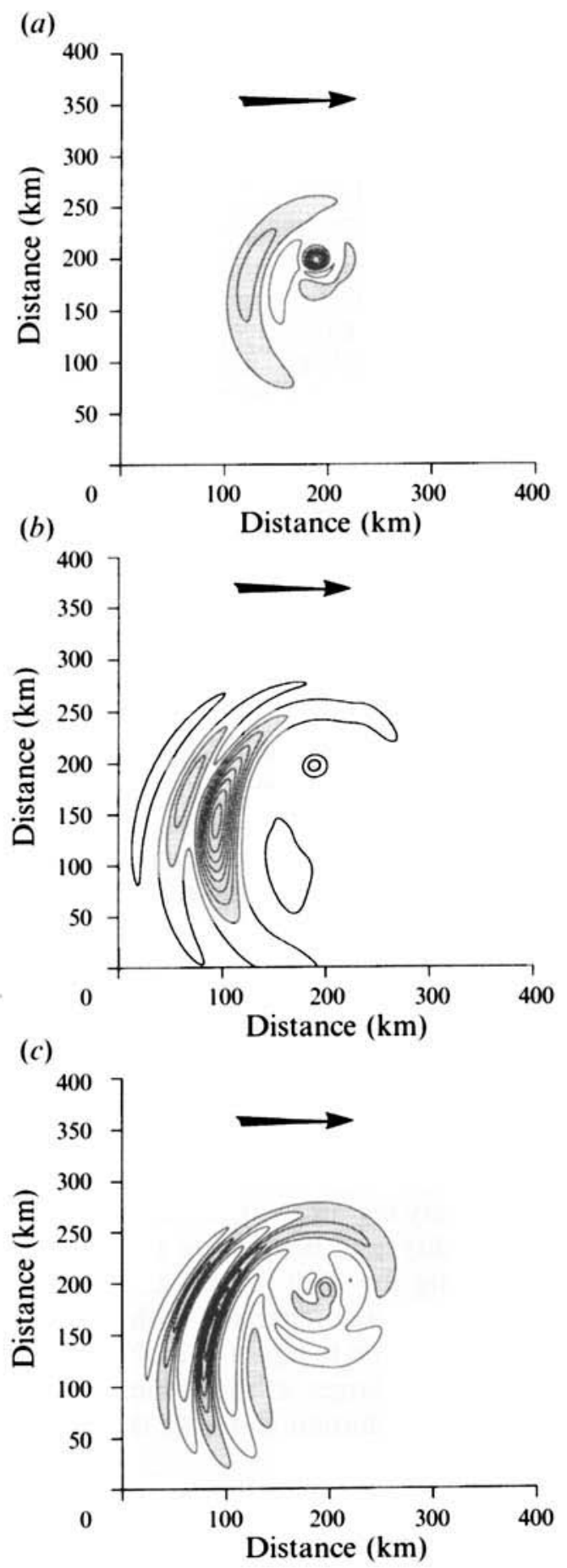

FIGURE 14. Experiment A1: kinetic energy (integrated over the mixed layer depth and averaged one inertial period), with the shaded area indicating energy higher than the background $\left(>0.11 \mathrm{~m}^{3} \mathrm{~s}^{-2}\right)$ : (a) at $t=10$ inertial periods, contour interval is $0.04 \mathrm{~m}^{3} \mathrm{~s}^{-2} ;(b)$ at $t=20$ inertial periods, contour interval is $0.1 \mathrm{~m}^{3} \mathrm{~s}^{-2}$. (c) Is mixed layer depth perturbation $h-h_{0}$ at $t=20$ inertial periods, with downwelling area shaded; contour interval is $2 \mathrm{~m}$, maximum is $11 \mathrm{~m}$. 
time. Later on the kinetic energy distribution within the eddy core follows the same time evolution as in the A0 experiment. On the other hand the kinetic energy maximum associated with the patch of inertial waves propagating upwind significantly increases. It attains the same value as the one within the eddy core at $t=12$ inertial periods and continues to increase while the latter keeps oscillating. Therefore after this time, the 'exponential-like' growth of the kinetic energy maximum displayed by the curve Al on figure 13 is related to the kinetic energy increase of this patch, which is entirely due to the instability mechanism. Within the last 10 inertial periods the kinetic energy maximum of this patch has grown by a factor 4.5 (figure $14 b$ ), resulting in an effective growth rate of $0.012 f$ for the r.m.s. velocity. Amplitude of the mixed-layer depth variations within the eddy is similar to the one displayed by A0 (less than $6 \mathrm{~m}$ ). However, the amplitude of the mixed-layer depth variations associated with the patch of waves propagating upwind increases from $3.6 \mathrm{~m}$ to $16 \mathrm{~m}$ within the last ten inertial periods (figure $14 \mathrm{c}$ ), leading to a growth rate of $0.0237 \mathrm{f}$. Note that the downwellings are more stiffened than the upwellings. As in most realistic cases involving a spectrum of lengthscales, those growth rates are smaller than the theoretical maximum value of $0.034 f$ corresponding to our parameters $\tau^{*}=0.24$ and $\nu^{*}=0.083$.

These results reveal that the inertial waves trapped within the anticyclonic eddy are weakly affected by the instability mechanism. Indeed the back and forth propagation of these waves (induced by the vorticity effects) completely inhibits the efficiency of the instability mechanism. On the other hand, as soon as a patch of inertial waves has been able to escape from the eddy area, the forced instability mechanism can work efficiently.

Let us examine now the situation with a cyclonic eddy, experiment $\mathrm{C} 1$. The time evolution of the kinetic energy distribution does not differ from experiment $\mathrm{C} 0$ during the first four inertial periods. Later on, the kinetic energy decrease near the eddy centre is larger in this experiment: the decrease attains a factor three (instead of two in the $\mathrm{C} 0$ experiment) within 20 inertial periods. As in $\mathrm{C} 0$, an energy maximum appears on the southwest edge of the negative vorticity ring (figure $15 a$ ). After about 10 inertial periods it slowly moves to the south outside the eddy area and its magnitude strongly increases (figure $15 b$ ): it is twice as big as the one in $\mathrm{C} 0$ experiment at $t=10$, and 3.5 times larger at $t=20$ inertial periods. The curve $\mathrm{Cl}$ in figure 13 is related to the time evolution of this maximum. As was the case in experiment A1, the inertial waves that have escaped from the eddy area and are propagating against the wind have an increasing amplitude owing to the forced instability (see figure $15 a$ and $b$ ). At $t=20$ inertial periods the amplitude of this path is not far from the maximum inside the eddy. The effective growth rate for its r.m.s. velocity is $0.013 f$ over the last 10 inertial periods, not far from the value found in experiment A1. On the other hand, the growth rate of the energy patch attached to the eddy is smaller (only $0.007 f$ ).

The most remarkable feature of the $\mathrm{C} 1$ experiment is the large $(36 \mathrm{~m})$ mixed-layer depth variations associated with small spatial scales on the east edge of the eddy core at $t=20$ inertial periods (figure $15 \mathrm{c}$ ). Small scales were present in experiment $\mathrm{C} 0$ (figure $10 b$ ) but their amplification in $\mathrm{Cl}$ results from the wind-forced instability. The large mixed-layer depth variations are associated with a secondary kinetic energy maximum that merges with the southwestern maximum during the last five inertial periods (figure $15 b$ ). This phenomenon has been found to be robust in a wide range of parameters (eddy vorticity, Rossby radius, wind stress).

This short analysis of the $\mathrm{Al}$ and $\mathrm{Cl}$ experiments reveals some resemblances and also significant differences between the anticyclonic and cyclonic eddy cases. In both cases, the inertial waves that have been expelled from the eddy and propagate against 

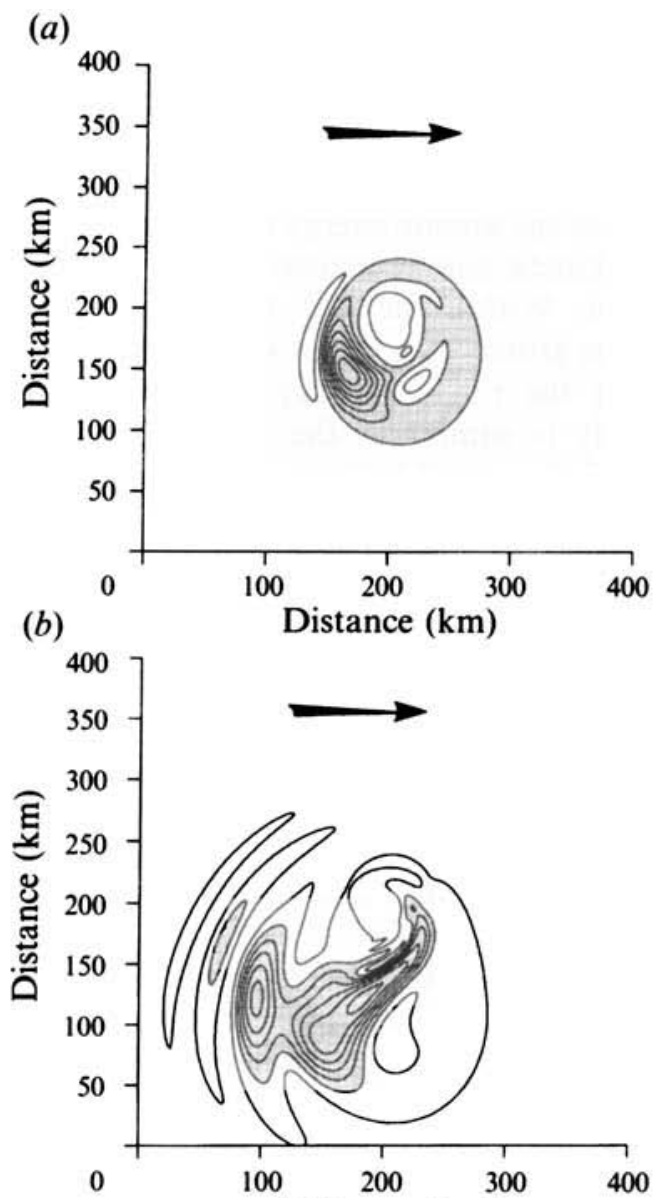

(c)

Distance (km)

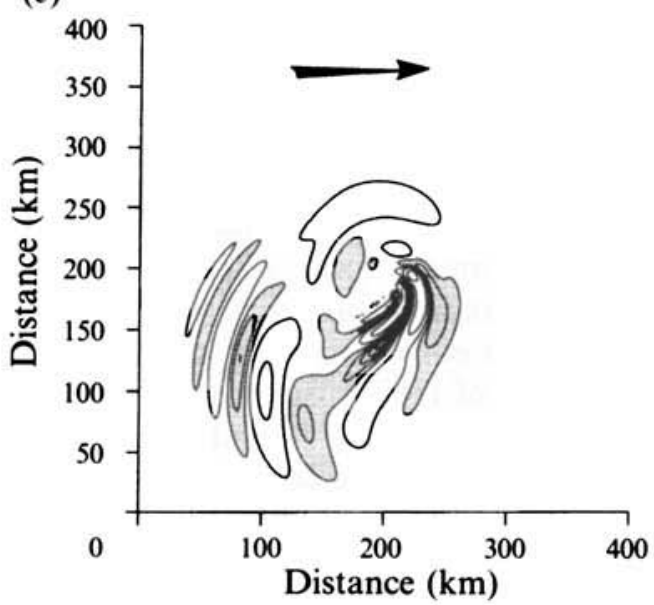

FIGURE 15. Experiment $\mathrm{Cl}$ : kinetic energy (integrated over the mixed layer depth and averaged one inertial period) with shaded area indicating energy higher than the background $\left(>0.11 \mathrm{~m}^{3} \mathrm{~s}^{-2}\right)$. $(a)$ At $t=10$ inertial periods, contour interval is $0.04 \mathrm{~m}^{3} \mathrm{~s}^{-2} ;(b)$ at $t=20$ inertial periods; contour interval is $0.1 \mathrm{~m}^{3} \mathrm{~s}^{-2}$. (c) Is the mixed-layer depth perturbation $h-h_{0}$ at $t=20$ inertial periods, with downwelling area shaded; contour interval is $3 \mathrm{~m}$, maximum is $30 \mathrm{~m}$. 
the wind grow. However, it should be noted that the expulsion of waves from the cyclonic eddy is less efficient than in the anticyclonic case as shown by the $\mathrm{A} 0$ and $\mathrm{CO}$ experiments. This explains the smaller kinetic energy values attained by these waves in the $\mathrm{Cl}$ experiment at $t=20$ inertial periods, compared with $\mathrm{Al}$. However, the dynamics of the inertial waves within the eddy is very different in $\mathrm{A} 1$ and $\mathrm{C} 1$. In the core of the anticyclonic eddy the instability mechanism is inhibited, as shown by the $\mathrm{A} 0$ and $\mathrm{A} 1$ experiments. In the cyclonic case the combination of the eddy dynamics and the forced instability generates a quite complex behaviour. There is a slight growth of the energy maximum, but also a large growth of small-scale upwellings and downwellings.

The efficiency of the instability mechanism has been assessed by running a large number of numerical experiments. A time-varying wind stress has the same effect as in $\S 3.1$. The energy level of the spatially uniform inertial oscillations in the far field is much higher, but the qualitative behaviour of the response and the amplification compared to the background are unchanged. A large decrease in energy is found from cases $\mathrm{A} 1$ and $\mathrm{C} 1\left(\tau^{*}=0.24\right)$ to cases $\mathrm{A} 2$ and $\mathrm{C} 2\left(\tau^{*}=0.2\right)$. This confirms the strong dependency of the growth rate (as the square of $\tau^{*}$ ) in dissipative regimes. Enhanced friction in experiments A3 and C3 $\left(v^{*}=0.33\right)$ lowers the energy level, but even in this very dissipative case there is still twice more kinetic energy in experiments forced by $\tau / h$ compared with experiments forced by $\tau / h_{0}$. Increasing the dissipation most dramatically affects the amplitude of the mixed-layer depth variations in the cyclonic eddy. At $t=20$, that amplitude is $6.6 \mathrm{~m}$ in experiment $\mathrm{C} 3$, compared with $36 \mathrm{~m}$ in $\mathrm{Cl}$.

\section{Conclusion}

The purpose of this paper was to investigate a new forced instability mechanism that affects inertial oscillations in the upper ocean mixed layer, allowing the waves propagating against the wind to extract energy and grow. This mechanism had first been examined by KT93 in a particular situation: wind blowing over a mesoscale onedimensional jet. We have shown here that this mechanism is far more general. The only required ingredients are a windstress acting for several inertial periods and an existing spatial variability of the inertial motions.

In a shallow-water model, the physics behind this mechanism are quite simple. The ratio $\tau / h$ of the wind stress and the mixed-layer depth produces an acceleration or deceleration of the mixed-layer velocity $u$. For inertial waves propagation against the wind, $h$ has a phase lag of $\pi$ relative to $u$ leading, over an inertial period, to a net kinetic energy flux from the wind to the inertial oscillations. Therefore, the instability happens in a shallow-water model only when the forcing is entered over the true depth $h$ of the mixed layer instead of the reference depth $h_{0}$.

An empirical formula for the maximum theoretical growth rate of the instability is

$$
\frac{\gamma_{m}}{f} \approx \frac{\tau^{* 2}}{16 v^{*}+2 \tau^{*}},
$$

with $\tau^{*}$ the non-dimensional wind stress and $\nu^{*}$ the non-dimensional dissipation. In the inviscid case, the maximum growth rate $\frac{1}{2} \tau^{*}$ is found in the limit of large wavenumbers. In the viscous case, inertial resonance leads to the selective amplification of horizontal scales close to the Rossby radius of deformation in a wide range of parameters.

The present paper was restricted, for simplicity, to the framework of shallow-water dynamics. KT93 have found the same instability in a fully stratified numerical model. In that case, the vertical advection of the momentum associated with the sheared 
Ekman velocities at the base of the mixed layer appeared to be the essential mechanism for instability, more than the variable mixed-layer depth $h$. This has yet to be confirmed by analytical calculations. Moreover, the fully stratified model shows that the rate at which energy leaves the mixed layer depends mainly on the stratification (Gill 1984). The equivalent $v$ is small when the inertial energy is confined to high vertical modes, which is the case when the sub-surface maximum in the Brunt-Väisälä frequency profile is sharp and shallow. A small mixed-layer depth $h_{0}$ and a small $\nu$ are achieved only in spring or summer conditions, when the seasonal thermocline is shallow and thin. Since high wind stress is found mainly in winter conditions, the instability of the inertial oscillations may happen only occasionally and locally in the ocean.

Our results show, however, that even when the growth rate is small $(\approx 0.02 f)$, the instability qualitatively modifies the behaviour of the wind-forced oceanic response. This has been demonstrated in the presence of a coast or mesoscale eddies, which both can generate variability at wavenumbers $k^{*}$ of order one. For coastal dynamics, the most striking result is the asymmetry between the (unstable) case of onshore winds and the (stable) case of offshore winds. In the presence of mesoscale eddies, instability can lead to energy maxima located outside the mesoscale structure and propagating against the wind. In both cases, the combination of instability and nonlinear advection leads to an enhanced amplitude of the mixed-layer depth variations.

The authors are supported by the Centre National de la Recherche Scientifique. We thank Mathieu Mory and Michel Crepon for giving us helpful comments on an early draft of the manuscript, and the anonymous reviewers for suggesting further improvements. Our investigation of the coastal problem was motivated by Michel Arhan's comments. Some calculations were performed at the Centre de Calcul Vectoriel pour la recherche in Palaiseau (France).

\section{REFERENCES}

ARHAN, M. 1973 Etude nonlineaire des ondes internes dans un milieu à deux couches fluides en rotation. Rapport CNEXO 17.

Day, C. G. \& WeBster, F. 1965 Some current measurements in the Sargasso sea. Deep-Sea Res. 12, $805-814$.

Crepon, M. 1969 Hydrodynamique marine en regime impulsionnel. Cah. Oceanogr. 21, 333-353.

CSanady, G. T. 1982 Circulation in the Coastal Ocean. Reidel. 279pp.

Gill, A. E. 1982 Atmosphere-Ocean Dynamics. Academic. 662pp.

Gill, A. E. 1984 On the behavior of internal waves in the wakes of storms. J. Phys. Oceanogr. 14, 1129-1151.

Grimshaw, R. 1988 Resonant wave interactions in a stratified shear flow. J. Fluid Mech. 190, 357-374.

KaMACHI, M. \& Grimshaw, R. 1984 Over-reflection of internal waves from the mixed layer. J. Fluid Mech. 141, 179-196.

KLein, P. \& HUA, B. L. 1988 Mesoscale heterogeneity of the wind driven mixed layer: influence of a quasi-geostrophic flow. J. Mar. Res. 46, 495-525.

Klein, P. \& Treguier, A. M. 1993 Inertial resonance induced by an oceanic jet. J. Phys. Oceanogr. 23, 1897-1915.

KunZE, E. 1985 Near-inertial wave propagation in geostrophic shear. J. Phys. Oceanogr. 15, 544-565.

Lelong, M. P. \& RiLeY, J. J. 1991 Internal wave-vortical mode interactions in strongly stratified flows. J. Fluid Mech. 232, 1-19.

Millot, C. \& CREPON, M. 1981 Inertial oscillations on the continental shelf of the gulf of LionsObservations and theory. J. Phys. Oceanogr. 11, 639-657. 
Niller, P. P. 1969 On the Ekman divergence in an oceanic jet. J. Geophys. Res. 74 (28), 7048-7052.

Pollard, R. T. 1970 On the generation by winds of inertial waves in the ocean. Deep-Sea Res. 17, 795-812.

Pollard, R. T. \& Millard, R. C. 1970 Comparison between observed and simulated windgenerated inertial oscillations. Deep-Sea Res. 17, 813-821.

Price, J. F. 1981 Upper ocean response to a hurricane. J. Phys. Oceanogr. 11, 153-175.

Renouard, D. P., Chabert D'Hières, G. \& Zhang, X. 1987 An experimental study of strongly nonlinear waves in a rotating system. J. Fluid Mech. 177, 381-394.

Treguier, A. M. \& HUA, B. L. 1987 Oceanic quasi-geostrophic turbulence forced by stochastic wind fluctuations. J. Phys. Oceanogr. 17, 397-411.

Weller, R. A. 1982 The relation of near-inertial motions observed in the mixed layer during the JASIN (1978) experiment to the local wind stress and to the quasi-geostrophic flow field. J. Phys. Oceanogr. 12, 1122-1136.

Weller, R. A., Rudnick, D. L., Eriksen, C. C., Polzin, K. L., Oakey, N. S., Toole, J. W., Schmitr, R. W. \& Pollard, R. T. 1991 Forced ocean response during the frontal air-sea interaction experiment. J. Geophys. Res. 96, 8611-8638. 